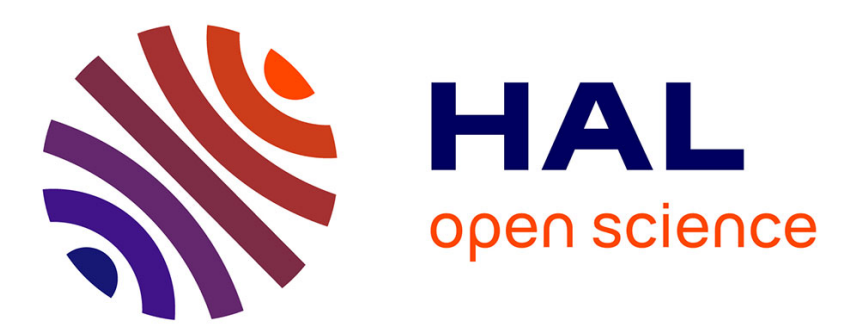

\title{
Comparison of Optimization Strategies for High-Lift Design
}

Pierluigi Iannelli, Frédéric Moens, Mauro Minervino, Rita Ponza, Ernesto Benini

\section{> To cite this version:}

Pierluigi Iannelli, Frédéric Moens, Mauro Minervino, Rita Ponza, Ernesto Benini. Comparison of Optimization Strategies for High-Lift Design. Journal of Aircraft, 2017, 54 (2), pp.642-658. 10.2514/1.C033648 . hal-01570145

\section{HAL Id: hal-01570145 \\ https://hal.science/hal-01570145}

Submitted on 28 Jul 2017

HAL is a multi-disciplinary open access archive for the deposit and dissemination of scientific research documents, whether they are published or not. The documents may come from teaching and research institutions in France or abroad, or from public or private research centers.
L'archive ouverte pluridisciplinaire HAL, est destinée au dépôt et à la diffusion de documents scientifiques de niveau recherche, publiés ou non, émanant des établissements d'enseignement et de recherche français ou étrangers, des laboratoires publics ou privés. 


\title{
Comparison of Optimization Strategies for High-Lift
}

\section{Design}

\author{
Pierluigi Iannelli* \\ CIRA - Italian Aerospace Research Centre, 81043 Capua, Italy
}

Frédéric Moens

ONERA - The French Aerospace Lab, 92190 Meudon, France

Mauro Minervino

Piaggio Aero Industries, 17038 Villanova d'Albenga, Italy

Rita Ponza ${ }^{\S}$, Ernesto Benini**

Università di Padova, 35131 Padova, Italy

\begin{abstract}
The design of high-lift systems represents a challenging task within the aerospace community, being a multidisciplinary, multi-objective and multi-point problem. The DeSiReH (Design, Simulation and Flight Reynolds Number Testing for Advanced HighLift Solution) project, funded by European Commission under the 7th Framework Program, aimed at improving the aerodynamics of high-lift systems by developing, in a coordinated approach, both efficient numerical design strategies and measurement techniques for cryogenic conditions. Within DeSiReH, different partners used several numerical automatic optimization strategies for high-lift system design purposes. A realistic multi-objective and multi-point optimization problem was defined and solved by adopting different flow models dimensionality, meshing techniques, geometry parameterization and optimization strategies. Special attention was devoted to perform a fair comparison of the results and useful information were obtained about trends, pros and cons of the approaches used. The outcome of these activities is that an efficient design
\end{abstract}

\footnotetext{
* Research Scientist, Fluid Mechanics Department, Aerodynamic Technologies and Icing Lab., p.iannelli@ cira.it

${ }^{\dagger}$ Research Engineer, Applied Aerodynamics Department, frederic.moens@onera.fr

* Research Engineer, former at Piaggio Aero Industries, now at CIRA: m.minervino@cira.it

$\S$ Research associate, Department of Industrial Engineering, r.ponza@ hit09.com

** Associate professor, Department of Industrial Engineering, ernesto.benini@unipd.it
} 
process can be set up through decoupling of the original multi-objective problem into several, sequential sub-optimization processes. Nevertheless, several decoupling possibilities exist and the most efficient one can be identified only on the bases of preanalysis or pre-knowledge of the specific problem. Secondly, the exercise carried out demonstrated the maturity and feasibility of a full 3D automatic high-lift design.

\section{Nomenclature}

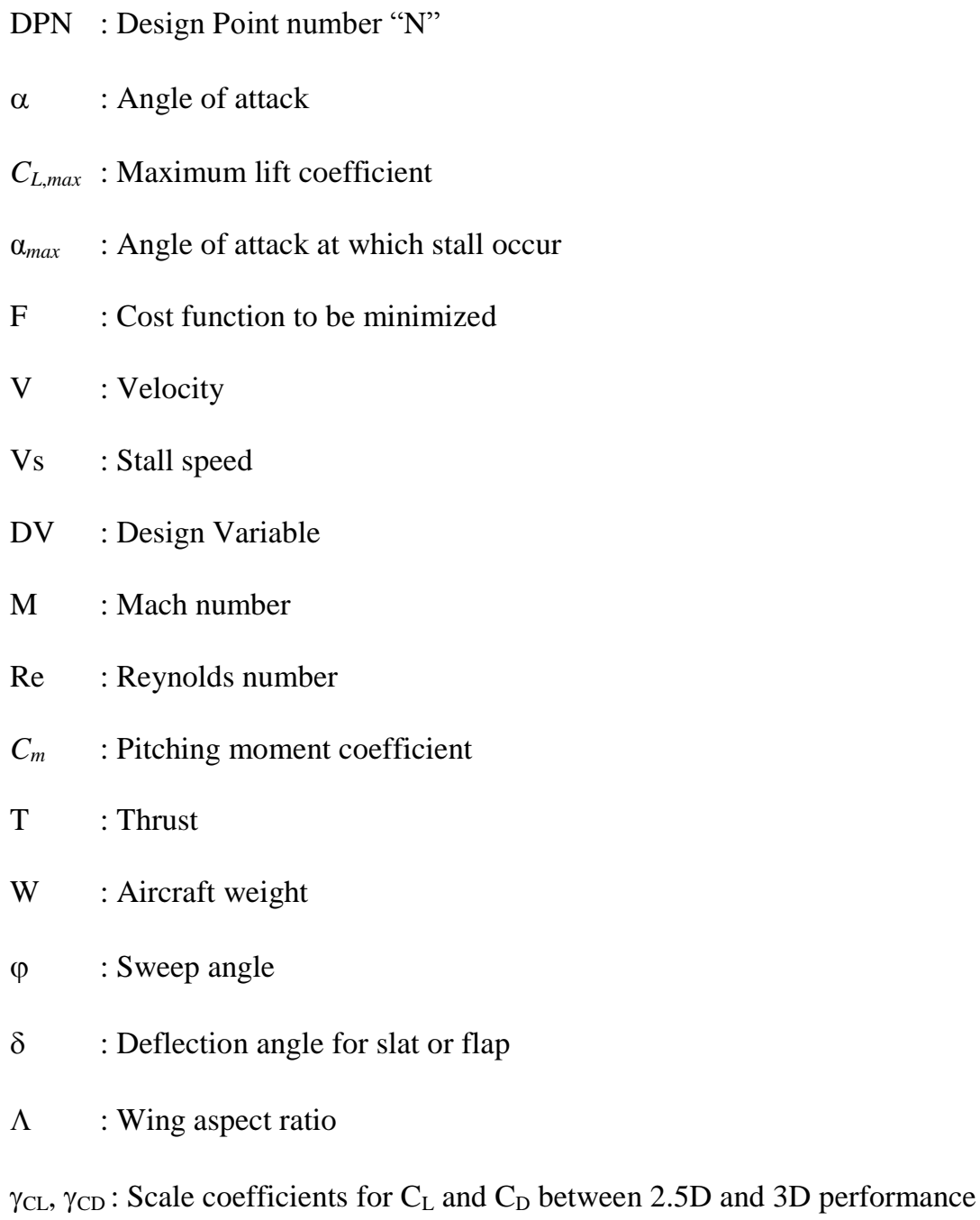

Partners involved:

AI-D : Airbus Deutschland (Germany)

AI-M : Airbus Military/EADS-CASA (Spain)

CIRA : Centro Italiano Ricerche Aerospaziali (Italian Aerospace Research Center)

DLR : Deutsches Zentrum für Luft- und Raumfahrt (German Aerospace Center)

ONERA : Office National d'Études et de Recherches Aérospatiales (French Aerospace Research Center) 
PAI : Piaggio Aero Industries (Italy)

UNIPD : Università di Padova (Italy)

\section{Acronyms:}

2D : Related to normal to leading-edge axis

2.5D : Related to stream-wise cut axis

3D : Related to aircraft

HL : High-lift

TO : Take-off

LDG : Landing

LE, TE : Leading edge, Trailing edge

CFD : Computational Fluid Dynamics

RANS : Reynold-Averaged Navier-Stokes

MOEAs : Multi-Objective Evolutionary Algorithms

CAD : Computer Aided Design

FAA : Federal Aviation Administration

A/C : Aircraft

MTOW : Maximum take-off aircraft weight

MLW : Maximum landing aircraft weight

OEW : Operating empty aircraft weight

SLS : Sea level static thrust

\section{Introduction}

$\mathrm{T}^{\mathrm{H}}$

HE design and optimization of high-lift (HL) systems has gained increasing importance over the last decades, especially considering their potential impact on aircraft sizing, costs and safety [1][2]. However, this is a quite challenging task for aircraft designers, due to its strong multidisciplinary, multi-objective and multi-point nature. In the design of HL devices, several performance indexes are to be improved, or at least controlled, at different flight conditions (e.g., take-off (TO), landing (LDG), approach, climbing, etc.). In fact, the design objectives are not only focused on achieving maximum lift requirements at TO and LDG [2], but also on achieving good performance in both the climb and approach phases while reducing both complexity and weight, thus resulting in a global cost reduction. Actually, HL systems may account for up to $10 \%$ of the production costs of a typical jet 
transport [3], due to the effort they require for being designed and tested, the inherent complexity of their actuation and support systems and the intensive maintenance they usually need. On the other hand, such efforts are justified by the fact that they make it possible to achieve reasonable runway lengths in TO and LDG of transport airplanes, while preserving their cruise efficiency.

Actually, even small improvements in HL systems aerodynamic design can produce significant gains in the aircraft overall weight and performance [4]. Leading edge (LE) devices such as slats are used for increasing the wing stall margin, while trailing edge (TE) devices such as flaps have the effect of shifting upwards the whole wing lift curve. In addition to their shape, the aerodynamic behavior of HL systems strongly depends on the settings of the different elements. Moreover, aerodynamic efficiency of HL devices must be guaranteed in multiple flight conditions, often featuring large aerodynamic loads over the various elements. Concerning the constraints of the HL design problem, a series of airworthiness requirements enforce considerable boundaries to the design space, often set the design point conditions and complicate the optimization problem formulation. In addition, both manufacturing and structural constraints must be included in the design problem definition from the very beginning (e.g., via specification of geometrical limitations such as trailing edges thickness, breakaway angles, etc.) in order to ensure that the designed shape can be manufactured and guarantees the structural stiffness needed to sustain the high aerodynamic loads occurring in the above mentioned conditions. Furthermore, the external shape of the high lift devices needs to be compliant with the designated cruise wing shape, when retracted in clean configuration. Finally, both mechanical integration and kinematical reliability aspects of the deployment system must be taken into account, such that unrealistic designs are avoided. All these features make the task of designing a HL system particularly difficult to be comprehensively tackled with a human based approach, due to both the large number of design variables involved and the large number (and different nature) of the constraints to be included, often giving rise to conflicting features and generating a narrow and sparse design space.

In the last decade, empirical approaches for analysis of HL multi-element configurations have been completely replaced by Computational Fluid Dynamics (CFD) tools (thanks also to the increased availability of computational resources), such that examples of CFD computations on multi-element airfoil are nowadays almost uncountable [2]. On the other hand, there are still some specific flow features difficult to be properly captured by CFD, such as multiple laminar/turbulent transitions [5], wakes interactions with boundary layers, and regions of flow detachment [6]. A quite recent review of CFD computations of HL systems is given in [7]. Prior to the widespread application of 2D Reynolds-averaged Navier-Stokes (RANS) methods (see [2] for a review), flows over multi- 
element airfoils were traditionally analyzed using inviscid and viscous flow solvers coupled in an interactive manner ([8]-[11]).

Moreover, aerodynamic optimization studies, including multi-objective methodologies, have been successfully performed. Actually, the HL system design is an application field where the large potentials and capabilities of automatic optimization can be successfully exploited to enhance the design quality and reduce the design cycle time (costs). In [12], a single-point method for optimizing the shape and position of high-lift system elements using an incompressible flow solver was illustrated: the objective was to maximize lift for a given drag at TO and LDG conditions. A gradient-based local optimizer was used in [13] for design optimization of a twodimensional aircraft high-lift system: in this case, flap shape functions and element positioning (deflection angle, gap, overlap) were considered as design variables for maximization of lift/drag ratio and single airfoil maximum lift coefficient.

In [14], an optimization procedure using the adjoint method is illustrated, where shape and deflection angles of the slat and flap elements are used for drag minimization and lift maximization subject to several constraints. Moreover, a Newton-Krylov (gradient-based) algorithm was used in [15] for the aerodynamic optimization of a multi-element airfoil: here the objective function sensitivities were calculated using an adjoint formulation with the purpose of lift enhancement and multipoint lift-constrained drag minimization. More recently, in [16] an aerodynamic design of high-lift multi-element airfoil was carried out based on a gradient-based optimization method using a 2D Navier-Stokes solver and sensitivity analysis method. In [17], a single-objective optimization based on a genetic algorithm was carried out for design optimization of both fixed-shape flap and slat settings in a three-element airfoil: in this case, the objectives of optimization were the minimization of drag at a given angle of attack and the separate maximization of aerodynamic efficiency at the same incidence. Flap and slat shape optimization including uncertainty of operating conditions was carried out in [18] using single-objective evolutionary algorithms coupled with a response surface methodology. Moreover, a recent, systematic CFD optimization study carried out with a genetic algorithm based design optimization procedure on a foil in water is described in [19]. Other known examples of design optimization studies can be found in the open literature [20]. Also, multi-objective approaches, especially those based on multi-objective evolutionary algorithms (MOEAs) have been applied to optimization of HL devices, in light of their capability to handle multi-objective design problems featuring highly nonlinear and multimodal functions. For instance, a multi-objective constrained optimization of multi-element airfoils is presented in [21], where the objective of optimization was the maximization of lift having as design variables those defining the flap setting. A further analysis is illustrated in 
[22], where different optimization strategies (including MOEAs), coupled with different types of flow solvers were used with the aim of improving lift and drag performance of a multi-element airfoil at TO through re-design of TE flap.

The issue of optimization of HL devices was one of the tasks in the DeSiReH project [23] (Design, Simulation and Flight Reynolds Number testing for advanced High Lift Solutions), funded by the European Commission within the $7^{\text {th }}$ Framework Program. Specifically, the project aimed at improving the aerodynamics of HL systems by considering, in a coordinated approach, the development of both efficient numerical design strategies and measurement techniques for cryogenic wind tunnel conditions. It represents the follow up to the EUROLIFT I and II projects [22], wherein both numerical and experimental studies mainly targeted validation of CFD tools for increasingly complexity configurations and up to flight Reynolds numbers.

Within this paper, an overview of the HL numerical optimization activities carried out within DeSiReH is presented. Firstly, a definition of an efficient formulations of the HL optimization problem and the identification of appropriate target functions (including airworthiness requirements), of reduced number of design variables for shape parameterization and cost function parameter evaluation were investigated and are presented in section II.

Section III of the paper presents the results obtained by different numerical optimization strategies based on high fidelity methods (CFD), for the design of high-lift systems. Within this "analysis" phase, a realistic optimization of the full HL flight envelope was addressed in a 2D/2.5D framework, by a group of partners adopting different approaches in terms of employed flow model, meshing techniques, geometry parameterization and optimization approaches/strategies. Moreover, a demonstration about the feasibility of a full 3D automatic optimization approach by employing industrial standards (from CAD to CFD) was addressed. The experience gained in the analysis phase was then exploited in another phase of $\mathrm{DeSiReH}$, wherein the design of an optimal feasible HL system has been developed for a high aspect ratio natural laminar wing, as detailed in [24]..

\section{Definition of the High Lift optimization problem}

Since both 2D and 3D optimization activities were planned, it was decided to define the optimization problem in a fully three-dimensional environment. The geometrical model assumed as starting point (baseline) for the optimization work is the 3D full span slat and flap DLR-F11 (KH3Y) wing-body HL configuration shown in Figure 1. A large experimental database is available for such 3D model from the former European project EUROLIFT. The 2D optimization work focused on the improvement of a wing section located in the middle of the outboard wing (Figure 1, right) where a 2D/2.5D flow assumption is more reasonable. 


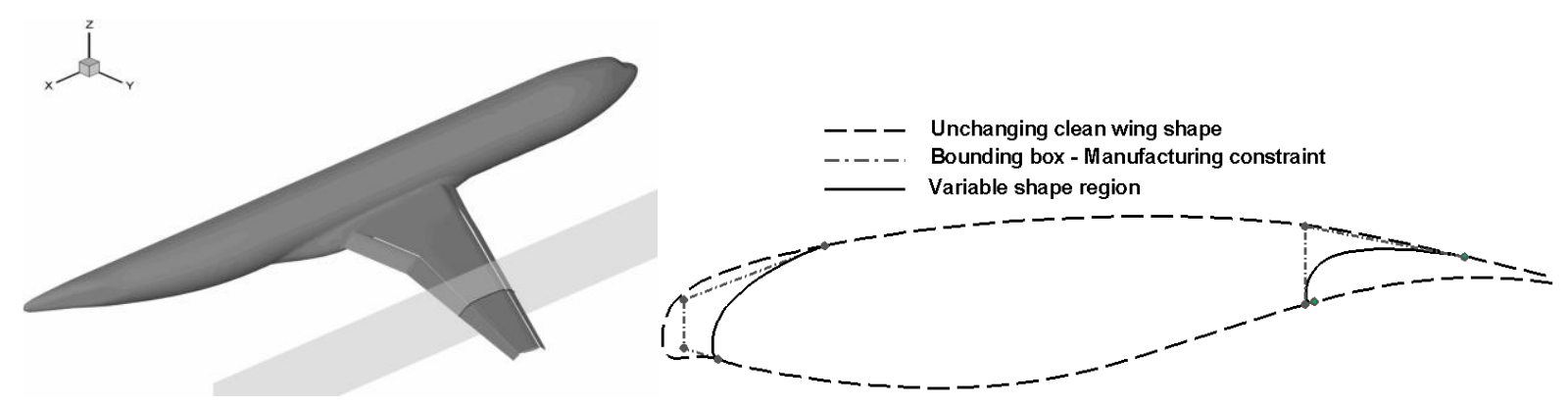

Figure 1: 3D KH3Y full span slat/flap model (left) and 2D cut at mid board of the outer wing (right)

\section{A. Design space}

As shown in Figure 1 (right), both slat and flap shape modifications were allowed only in the solid line regions and were constrained by both aft and rear 3D polygonal bounding boxes. Such bounding boxes were introduced to mimic the manufacturing limitations introduced by both the slat and main wing spar positions and by breakaway angles (related to minimum thickness requirements at elements' trailing edges), respectively. In order to limit the design space extent, a number of assumptions were made about the allowed geometrical modifications:

- Elements' positioning had to be optimized, e.g. slat and flap deployment angle, gap and overlap.

- Shape modifications were allowed within the bounding boxes shown in Figure 1 (right).

- Slat and flap chord, stat trailing edge thickness, shroud trailing edge thickness and wing shroud trailing edge position were assumed to be fixed.

Moreover, in order to leave enough margins for improvements, it was decided to avoid the introduction of any kinematical constraining rule, which would introduce limitations on the allowed elements' displacement.

\section{B. Optimization targets and design points}

Regarding HL system performance improvement possibilities, a wide list was prospected after a review of all the possible performance indexes relevant to the several flight phases in which the HL system is employed (e.g., takeoff, landing, approach, climb, etc.) Among those available, it was chosen to focus the optimization work towards the following two targets:

1. Reduction of time-to-climb in the $2^{\text {nd }}$ climb segment at take-off.

2. Reduction of fuel burn in the final landing approach phase.

It is worth noting that both objectives are related to drag reduction purposes, which is also beneficial from the pollution emissions reduction point of view, both in terms of chemicals and aerodynamic noise. Moreover, the consortium partners agreed not to target a maximum lift coefficient $\left(C_{L, \max }\right)$ improvement for two main reasons. 
The first being the huge amount of computational resources required in a full 3D simulation environment, especially in cases where no automatic $C_{L, \max }$ calculation capabilities are available. The second reason being that a large part of the optimization work was carried out by using a 2D approach, and the selected wing section is not representative of the three dimensional stall phenomenon occurring on the whole wing.

The FAA airworthiness requirements for commercial transport aircrafts (CFR14 - Part 25) fix the minimum speeds relevant to the two flight conditions referred in the objectives above to $V_{2}=1.13 V_{S}$ and $V_{R E F}=1.23 V_{S}$ for TO and LDG, respectively ( $V_{S}$ being the relevant stall speed). According to this, a first design point (DP1) associated to each of the above objectives could be specified in terms of $3 \mathrm{D}$ incidences $\left(\alpha_{3 D, \mathrm{TO}}=11.91^{\circ}\right.$ and $\alpha_{3 D, \mathrm{LDG}}=6.54^{\circ}$, respectively), as extracted from the experimental curves of the baseline configurations shown in Figure 2.
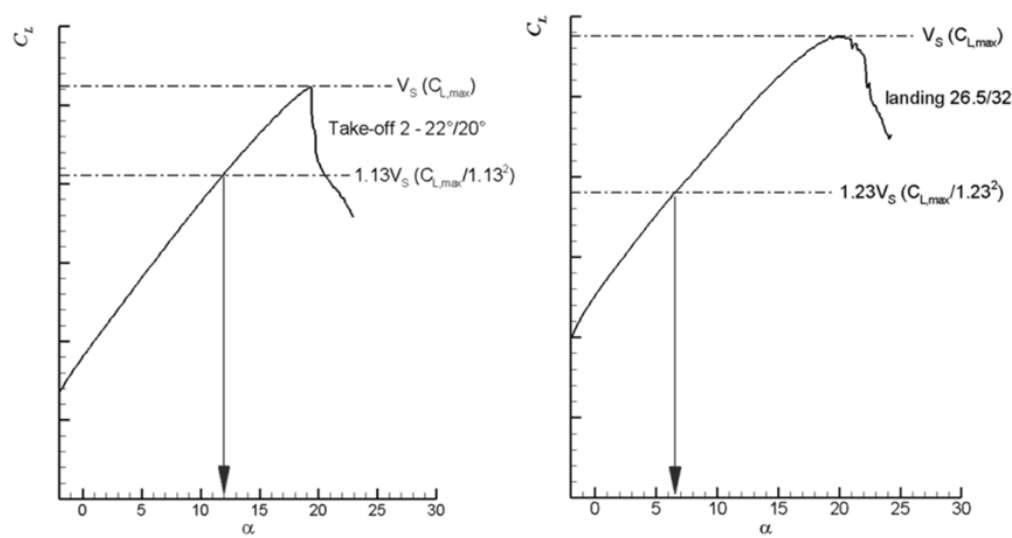

Figure 2: Experimental 3D lift curves of TO (left) and LDG (right) configurations. $\left(M=0.2, \operatorname{Re}=15 \times 10^{6}\right)$.

The above design points were defined in the linear part of the lift curves, therefore an additional design point (DP2) was added in order to control the lift curve behavior of the optimized configuration close to maximum lift conditions, i.e. at $\alpha_{3 D}=17^{\circ}$ for both TO and LDG, as detailed in the following. Based on the above considerations, the design points considered in the optimization are four, two for the TO optimization problem and two for the LDG one:

$$
\begin{aligned}
& \text { TO - DP1 : } M_{\infty}=0.2, \mathrm{Re}_{\infty}=15 \times 10^{6}, \alpha_{3 D}=11.91^{\circ} \\
& \text { TO - DP } 2: M_{\infty}=0.2, \mathrm{Re}_{\infty}=15 \times 10^{6}, \alpha_{3 D}=17^{\circ} \\
& \text { LDG - DP1 : } M_{\infty}=0.2, R \mathrm{e}_{\infty}=15 \times 10^{6}, \alpha_{3 D}=6.54^{\circ} \\
& \text { LDG - DP } 2: M_{\infty}=0.2, \mathrm{e}_{\infty}=15 \times 10^{6}, \alpha_{3 D}=17^{\circ}
\end{aligned}
$$

\section{Aerodynamic and airworthiness constraints}

In general, several constraints have to be included in an HL optimization problem statement. They are normally based on different requirements coming from other disciplines like, e.g. aerodynamics, airworthiness, kinematics feasibility, structural reliability, etc. While the structural requirements were somehow defined by means of the 
bounding boxes described in section A, a first couple of aerodynamic constraints are directly associated to the DP1 of both configurations, since the optimized configuration was required to provide at least the same lift level of the baseline configuration $\left(C_{L}\right.$ being the 3D lift coefficient):

$$
\left.C_{L}\right|_{@ \mathbf{T O}-\mathbf{D P 1}} \geq\left. C_{L, \text { initial }}\right|_{@ \mathbf{T O}-\mathbf{D P 1}} ;\left.\quad C_{L}\right|_{@ \mathbf{L D G}-\mathbf{D P 1}} \geq\left. C_{L, \text { initial }}\right|_{@ \mathbf{L D G}-\mathbf{D P 1}}
$$

As stated above, in order to ensure a proper behavior of the lift curve at high incidence (DP2 points), it was required that the optimized configuration ensures lift levels equal to or higher than the baseline:

$$
\left.C_{L}\right|_{@ \mathbf{T O}-\mathbf{D P} 2} \geq\left. C_{L, \text { initial }}\right|_{@ \mathbf{T O}-\mathbf{D P} 2} ;\left.\quad C_{L}\right|_{@ \mathbf{L D G}-\mathbf{D P} 2} \geq\left. C_{L, \text { initial }}\right|_{@ \mathbf{L D G}-\mathbf{D P} 2}
$$

Furthermore, due to the usage of a non-strict requirement on $C_{L, \max }$, an a posteriori verification check was foreseen for the maximum lift capabilities of the optimum configurations. Accordingly, two verification points were defined at $\alpha=19^{\circ}$ and $\alpha=20^{\circ}$ for TO and LDG, respectively:

$$
\left.C_{L}\right|_{\mathbf{T O} @ \alpha=19^{\circ}} \geq\left. C_{L, \text { initial }}\right|_{\mathbf{T O} @ \alpha=19^{\circ}} ;\left.C_{L}\right|_{\mathbf{L D G} @ \alpha=20^{\circ}} \geq\left. C_{L, \text { initial }}\right|_{\mathbf{L D G} @ \alpha=20^{\circ}}
$$

For the 2D activities, the post-check in Eq. (4) was performed by considering the 2D force coefficients. Finally, an additional aerodynamic constraint was introduced on the (either 2D or 3D) pitching moment coefficient $C_{m}$, which was allowed to increase by a $15 \%$ margin with respect to the baseline configuration (such a large margin was agreed in order to leave enough design space for optimization):

$$
-C_{m 25} \leq-1.15 \cdot C_{m 25}^{\text {initial }}
$$

Such constraint had to be respected at all design points defined above.

Among others, two constraints for the LDG phase come from airworthiness requirements on the minimum thrust level to be held during the final approach phase, and the minimum climb gradient in the go-around phase, as follows. Regarding the approach, the only common phase between all possible approaches (depending on aircraft type, airport characteristic, approach strategy, etc.) is the last segment within the 5 Nautical Miles range, where the $\mathrm{A} / \mathrm{C}$ has to be trimmed on a $3^{\circ}$ glide slope at $V_{R E F}=1.23 V_{S}$ and at a thrust level higher than approach idle. This condition translates into the following constraint:

$$
T_{A P} \leq T=\left.\left.W\left[\frac{C_{D}}{C_{L}}-\sin 3^{\circ}\right]\right|_{V=V_{R E F}=1.23 \cdot V_{S}} \Rightarrow \quad C_{D}\right|_{@ \mathbf{L D G}-\mathbf{D P 1}} \geq 0.045 \cdot C_{L, \max }
$$


where $T$ and $T_{A P}$ indicate thrust and thrust at approach idle, respectively, and $W$ is the $\mathrm{A} / \mathrm{C}$ weight. To derive Eq. (6), the idle thrust to weight ratio $\left(T_{A P} / W\right)$ is needed at landing conditions. This value was derived from an analysis regarding all transport aircraft data available (shown in Figure 3 for the maximum take-off weight), which shows a clear trend for the static thrust vs. weight and an unexpected similarity between all aircrafts.

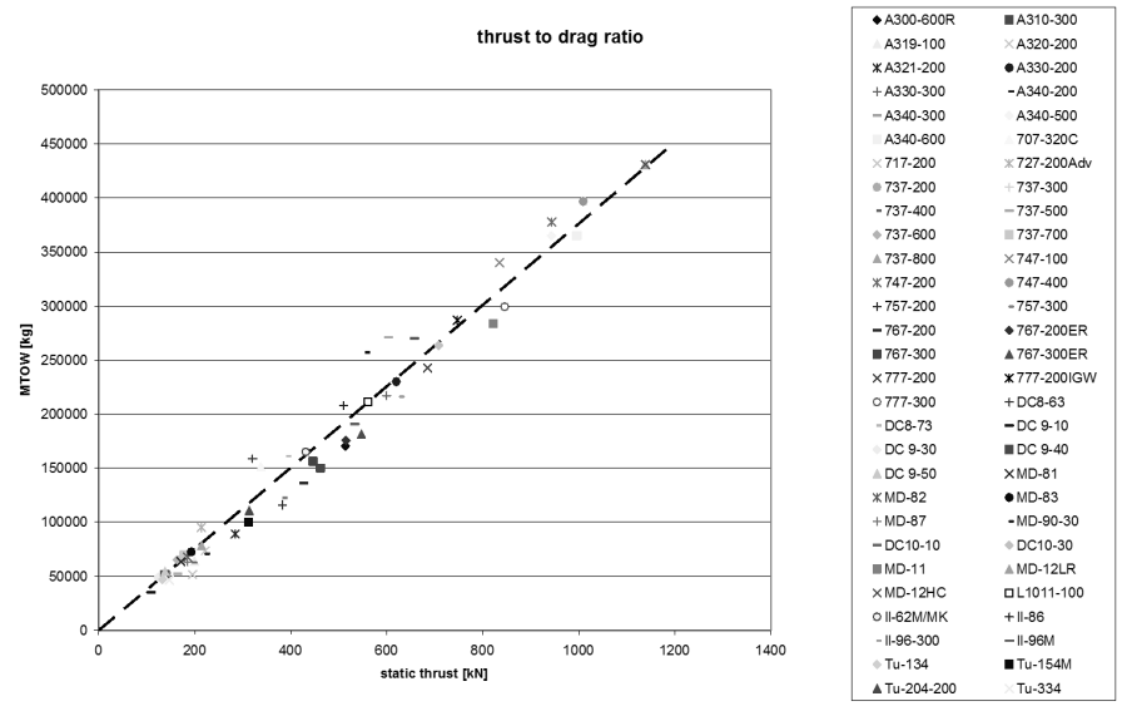

Figure 3: Example of available civil aircraft data. MTOW vs. static thrust at sea level.

From these data, the following quantities were derived via 0.999-regression analysis:

$$
\frac{T_{S L S}}{M T O W}=0.271 \pm 0.01 ; \frac{T_{S L S}}{M L W}=0.368 \pm 0.015 ; \frac{T_{S L S}}{O E W}=0.566 \pm 0.037
$$

where $T_{S L S}$ is the sea level static thrust and $M T O W, M L W, O E W$ are the maximum take-off, maximum landing and operating empty weight, respectively. Moreover, in deriving Eq. (6) the following relations between $T_{S L S}$, cruise thrust $T_{C R}$ and $T_{A P}$ were considered:

$$
T_{A P}=0.25 \cdot T_{C R}, \quad T_{C R} \approx 0.175 \cdot S L S T
$$

Regarding the go-around phase, the FAR regulations pose a restriction on the minimum climb path, which is expressed as a constraint:

$$
\sin \gamma=\left[\frac{T}{G}-\frac{C_{D}}{C_{L}}\right]_{V=V_{R E F}=1.23 V_{S}} \geq\left. 3.2 \% \Rightarrow C_{D}\right|_{@ \mathbf{L D G}-\mathbf{D P 1}} \leq 0.222 \cdot C_{L, \max }
$$

\section{Overview of the optimization problem and analysis of objective functions}

A synthetic view of the bi-objective/multi-point optimization problem formulated from previous sections, entirely defined in a 3D framework, is summarized in Figure 4. 

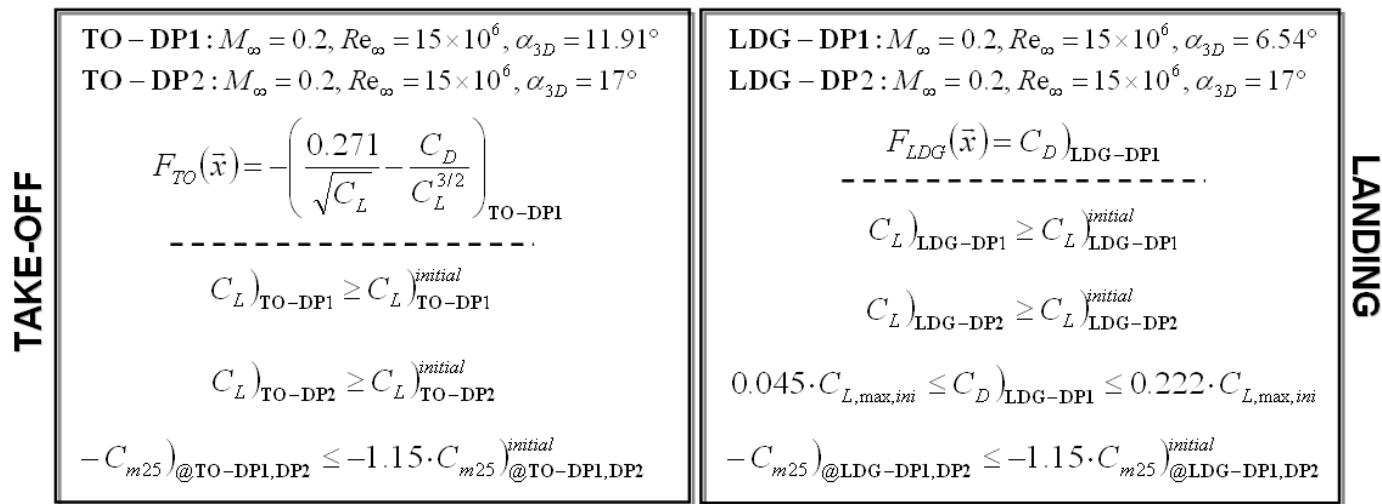

Figure 4: Overview of the bi-objective/multi-point optimization problem.

In the optimization problem statement reported in Figure $4, F_{T O}$ and $F_{L D G}$ are the TO and LDG objective functions, respectively, whereas $\bar{x}$ indicates the vector of design variables (DVs). For the case at hand, the vector $\bar{x}=\left[\bar{s}, \bar{p}_{T O}, \bar{p}_{L D G}\right]$ contains the flap and slat shape design variables vector $\bar{s}$, the TO slat/flap position design variables vector $\bar{p}_{T O}$ and the LDG slat/flap position design variables vector $\bar{p}_{L D G}$.

It is worth remarking that in order to reduce the huge computational requirements posed by the optimization problem considered, the partners working with 3D CFD optimization only considered the left part of the problem, i.e. only the TO optimization problem, and only the 3D flap shape and setting optimization was required.

One peculiarity of the proposed design problem is that the design variables contained in the vector $\bar{s}$ affect in principle both TO and LDG objective functions, whereas the DVs in vector $\bar{p}_{T O}$ affect only $F_{T O}$ and the DVs in vector $\bar{p}_{L D G}$ affect only $F_{L D G}$. Alternatively, one can say that two objectives are linked by means of the sole slat/flap shape variables, whereas slat/flap positioning variables affect either the TO or the LDG objective only. One purpose of the proposed exercise was to verify if the link between the two objectives is strong or if the shape variables $\bar{S}$ affect mainly only one of the two objectives (weak coupling of the objectives). In the first case the outcome would indicate that for such kind of problems there is a need for a coupled optimization which must include both TO and LDG objectives at the same time, whereas in the case of a weak coupling the usage of a simpler sequential optimization strategy (e.g., LDG first, TO then) might be considered.

Prior to the optimization work, a sensitivity study was performed for a range of lift and drag variations in order to get useful information about the potential improvements of the objective functions. By using the available experimental $C_{L}$ and $C_{D}$ values of the initial configuration, the plot shown in Figure 5 was drawn, showing that for a fixed drag level (corresponding to a specific curve in the plot), the TO objective function increases with $C_{L}$. 


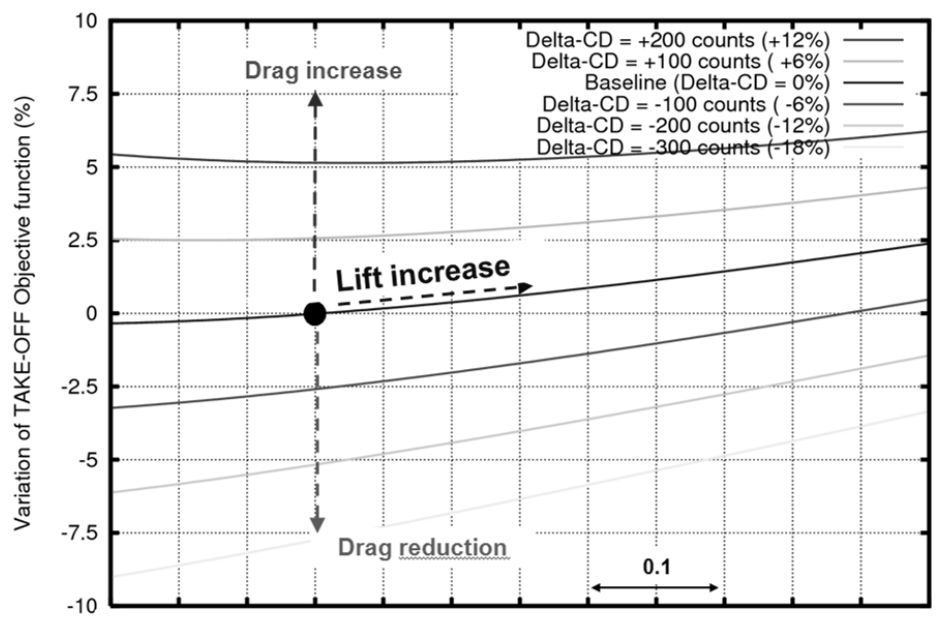

$\mathrm{CL}$

Figure 5: Parametric analysis of TO objective function.

According to Figure 5, a first observation is that the lift coefficient should be kept as much as possible close to the baseline value to maximize the potential objective function reduction. Similarly, for a given drag reduction, the maximum potential TO objective function improvement (reduction) is achieved with a lift value equal to the baseline (lower values of lift are not allowed by the lift constraint). Concerning the amount of drag reduction, the plot shows that at $C_{L}=C_{L \text {,baseline }}$, a $6 \%$ reduction in $C_{D}$ implies only approximately $2.5 \%$ reduction in the overall TO objective function. However, it is worth noting that a $6 \%$ reduction in $C_{D}$ means 100 drag counts (i.e., 0.01) reduction with respect to the 3D baseline, which would be quite an ambitious target for the already optimized HL configuration under consideration. Therefore, if we assume a target $C_{D}$ reduction at TO of about 50 drag counts, it would give a TO objective reduction of approximately $1.25 \%$, which is the order of magnitude of the expected improvements at TO. Similar considerations can be drawn at LDG, though in this case a $6 \%$ reduction in $C_{D}(\approx 100$ drag counts) would reduce by the same amount the LDG objective function. Accordingly, both TO and LDG problems basically reduce to a drag minimization problem at constant lift. On the other hand, it must be also observed that a lift constraint violation, if not treated either explicitly or by means of a step penalty, can easily lead to misleading results if suitable penalty functions are not supplied to the optimizer. As a matter of fact, a $C_{L}$ reduction would introduce a reduction in induced drag, which would produce a fake objective functions improvement. To this aim, suitable penalty function expressions have been derived in [25] for both TO and LDG problems, in order to properly balance the possible artificial improvements coming from lift constraints violations. With similar considerations, the derivation of RANS simulations convergence criteria have been derived in [25]. In fact, as well known, in RANS computations it can happen that while the equations' residuals drop significantly, the aerodynamic force coefficients still oscillate within a certain range during the iterative solution process. On 
the other hand, within an optimization loop requiring huge computational resources, it is fundamental to limit the number of iterations per simulation. Therefore, forces convergence criteria are needed, in order to terminate the computation as quick as possible though without introducing too much uncertainty (noise) on the overall objective function estimation.

\section{3D AERO-PERFORMANCE PREDICTION BASED ON A 2D SIMULATION}

As stated above, both three-dimensional and two-dimensional optimization activities were considered . While for $3 \mathrm{D}$ activities the aerodynamic performance prediction is straightforward, a specific strategy was employed to predict the $3 \mathrm{D}$ performance based on a simple $2 \mathrm{D}$ or $2.5 \mathrm{D}$ flow simulation. For the two-dimensional activities the starting geometry is represented by the (2.5D) stream wise cut of the wing, as shown in Figure 1. In the following sections it is described the strategy used to perform the elements deployment, the flow and geometry normalization, the flow solvers calibration and the prediction of 3D Wing-Body performance based on 2Dnormalized CFD results.

\section{E. Deployment of high-lift elements in 2D}

In the deployment of a real 3D HL device, it must be taken into account that each element rotation occurs with respect to a swept rotation axis, which can be assumed parallel to:

- $\quad$ the fixed leading edge for the slat, and;

- $\quad$ the shroud trailing edge for the flap.

The pure $2 \mathrm{D}$ rotation of a given HL device into the stream wise plane would lead to an imprecise geometry description, whose extent will depend on the magnitude of the wing sweep angle. Instead, any device rotation should be first performed in a coordinate system normal to the relevant aforementioned swept rotation axis, and then the corresponding geometry should be re-projected onto the stream wise plane. To this aim, the following transformations have been derived):

$$
\begin{aligned}
& X(\delta)=X_{0}+\left(X-X_{0}\right) \cdot \cos \delta-\frac{\left(Y-Y_{0}\right)}{\cos \varphi} \cdot \sin \delta+\Delta X \\
& Y(\delta)=Y_{0}+\cos \varphi \cdot\left(X-X_{0}\right) \cdot \sin \delta+\left(Y-Y_{0}\right) \cdot \cos \delta+\Delta Y
\end{aligned}
$$

In equation (10), $\varphi$ is sweep angle of the rotation axis and $\delta$ is the deflection angle assigned to the slat/flap element. Moreover, $(X(\delta), Y(\delta))$ are the coordinates of a point in the stream wise (2.5D) plane consequent to a rotation $\delta,(X, Y)$ are the coordinates of a point in the stream wise plane prior to the rotation, $\left(X_{0}, Y_{0}\right)$ represent the 
rotation point in the stream wise plane. Finally, $(\Delta X, \Delta Y)$ represent an assigned translation in the stream wise plane.

Suitable values of $\varphi, \delta,\left(X_{0}, Y_{0}\right)$ and $(\Delta X, \Delta Y)$ have to be pre-selected in order to define the deflected initial TO and LDG 2.5D geometries. By starting with the stream wise cut of the retracted (clean) configurations, such values were initially calibrated in order to obtain deployed geometries matching the deployed stream wise cuts extracted from the relevant 3D TO and LDG CAD models, respectively. An example of calibration for the TO case is presented in Figure 6, wherein by starting with the 2.5D cut of the clean configuration, the elements have been deployed according to Eq. (10) and the resulting geometry is compared with the stream wise cut of the 3D HL wing. In the proposed optimization problem, $\delta$ and $(\Delta X, \Delta Y)$ represent the setting variables to be optimized for both slat and flap at TO and LDG conditions.

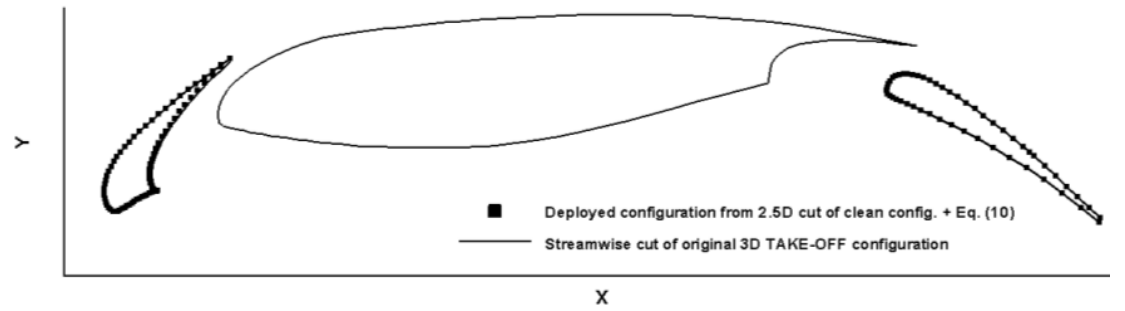

\section{Figure 6: Deployed TO configuration. Stream wise cut from 3D CAD model (line) vs. deployed 2D configuration (symbols) according to Eq. (10).}

\section{F. Geometry and flow variables normalization, calibration of 2D flow simulation}

After the deployment strategy defined by Eq. (10), the aerodynamic performance are evaluated by means of either a 2.5D flow computation or a pure 2D flow simulation, though in the last case a 2D-normalized airfoil geometry needs to be considered. The 2D-normalized airfoil, whose coordinates are indicated with $\left(x_{2 D-n o r m}, y_{2 D}\right.$ norm) is derived by projecting the streamwise (so called 2.5D) wing section onto a plane orthogonal to the reference sweep chosen $\varphi_{\text {norm }}$ (e.g., clean wing leading edge sweep, fixed leading edge sweep, etc..):

$$
x_{2 D-n o r m}=X ; \quad y_{2 D-n o r m}=\frac{Y}{\cos \varphi_{\text {norm }}}
$$

Moreover, when performing flow calculations in the 2D-normalized reference system, the inflow conditions, Mach and Reynolds numbers, respectively, are specified according to the following relations:

$$
M_{2 D-n o r m}=M_{3 D} \cos \varphi_{\text {norm }} ; \operatorname{Re}_{2 D-n o r m}=\operatorname{Re}_{3 D} \cos ^{2} \varphi_{\text {norm }}
$$


Accordingly, the forces in the 2D-normalized plane (pressure, lift and drag coefficients) are related to the stream wise plane ones (2.5D) by means of:

$$
c_{p_{2 D-n o r m}}=\frac{c_{p_{2.5 D}}}{\cos ^{2} \varphi_{\text {norm }}} ; c_{l_{2 D-n o r m}}=\frac{c_{l_{2.5 D}}}{\cos ^{2} \varphi_{\text {norm }}} ; c_{d_{2 D-n o r m}}=\frac{c_{d_{2.5 D}}}{\cos ^{2} \varphi_{\text {norm }}}
$$

During an initial code calibration phase, the 2D-normalized pressure coefficient obtained by CFD were compared to the available 3D sectional experimental data, in order to find suitable values for both the sweep angle $\varphi_{\text {norm }}$ and the angle of attack $\alpha_{2 D-n o r m}$. Specifically, for each 3D design point flow conditions (cf., Eq.(1)), a relevant angle of attack $\alpha_{2 D \text {-norm }}$ was determined. These values are those to be used within a $2 \mathrm{D}$-normalized computation, in order to have a reasonable similarity between the simulated 2D flow and the real 3D flow behavior occurring when the same wing section is working within a full 3D Wing-Body flow. As an example, Figure 7 shows a comparison between the experimental sectional pressure distribution measured on the $3 \mathrm{D}$ wing and the pressure distribution obtained from a CFD computation after calibration. In this case the sweep angle $\varphi_{\text {norm }}$ has been calibrated in such a way to match the experimental stagnation pressure coefficient value at the flap leading edge, whereas the value of $\alpha_{2 D-n o r m}$ in computations was calibrated in such a way to get a best-fit of the experimental sectional pressure distribution at slat and main wing leading-edges.

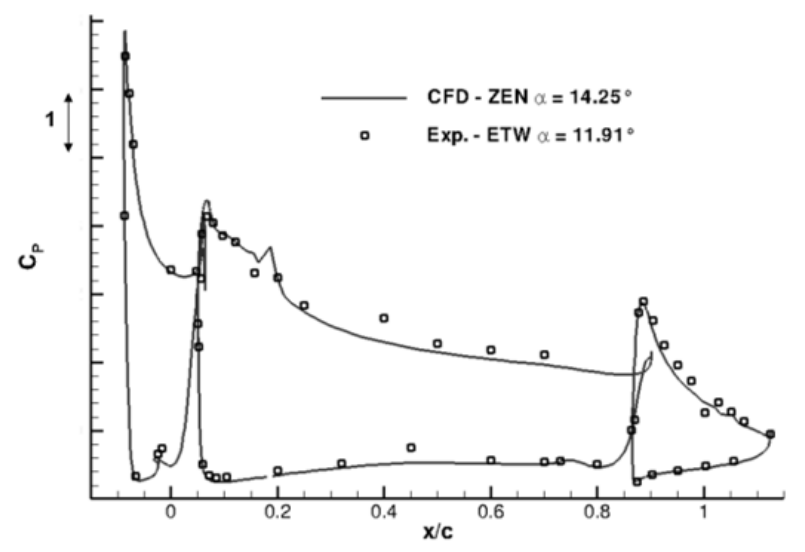

\section{Figure 7: Comparison of sectional pressure distribution between 3D experiment and 2D-normalized CFD results from CIRA's flow solver ZEN.}

Thanks to such a procedure, there is a well-defined relation between each 3D incidence $\alpha_{3 D}$ of the Wing-Body polar and the corresponding angle of attack $\alpha_{2 D-n o r m}$ to be used within a 2D-normalized computation. Similarly is for partners working with 2.5D flow simulations.

As it will be highlighted in the following, no experimental aerodynamic force data was available for the bidimensional wing section considered herein, since only 3D force data have been collected in the experimental campaign. Therefore, despite the achieved flow similarity in terms of pressure coefficient distribution, no 
calibration was possible between the computed 2D force coefficients and the experimental sectional force coefficients.

\section{G. Prediction of 3D wing performance based on 2D-normalized CFD results}

An approximate method was used to predict the overall 3D wing behavior based on 2D results, which was already successfully employed in ref. [22]. It is based on the following approximation hypothesis:

- The overall shape of the span wise lift and drag distribution of the 3D wing is not changed by local modifications of the slat/flap in the selected wing section. In other words it is assumed that any modification in the selected wing section is proportionally propagated throughout the whole wingspan.

- The span wise lift and drag distribution of the 3D wing can be scaled based on the lift and drag changes of the selected 2.5D wing section.

Such assumptions are reasonable for the wing type considered herein, since it incorporates full-span slat and flap devices. The correlation between the stream wise airfoil (2.5D) and 3D wing performance were found by comparisons of calculated 2D section data and experimental measurements available for the 3D HL model considered herein. The following relations apply:

$$
c_{L_{3 D}}=c_{l_{2.5 D}} \cdot \gamma_{C_{L}} \quad ; \quad c_{D_{3 D}}=\gamma_{C_{D}}\left(c_{d_{2.5 D}}+c_{l_{2.5 D}}^{2} / \pi \tilde{\Lambda}\right)
$$

where $\gamma_{C_{L}}$ and $\gamma_{C_{D}}$ are two correlating scaling factors, $\Lambda$ is the wing aspect ratio and $\tilde{\Lambda}=\Lambda \cdot \gamma_{C_{D}} / \gamma_{C_{L}}^{2}$ is a modified aspect ratio. As an example, Figure 8 shows how the 2D-normalized lift and drag coefficients correlates with the 3D experimental data through usage of Eq. (14). The calibrated numerical procedure described is able to properly capture not only the salient 3D flow features occurring on the analyzed wing section, but it is also able to reasonably predict the overall 3D wing performance by means of a low-cost $2 \mathrm{D}$ simulation. However, due to the intrinsic three-dimensional nature of the wing stall phenomenon, the above procedure is unsuitable in predicting the aerodynamic performance close, at and beyond stall conditions. This happens also because the wing section considered herein is not representative of the stall onset on the real $3 \mathrm{D}$ wing. 

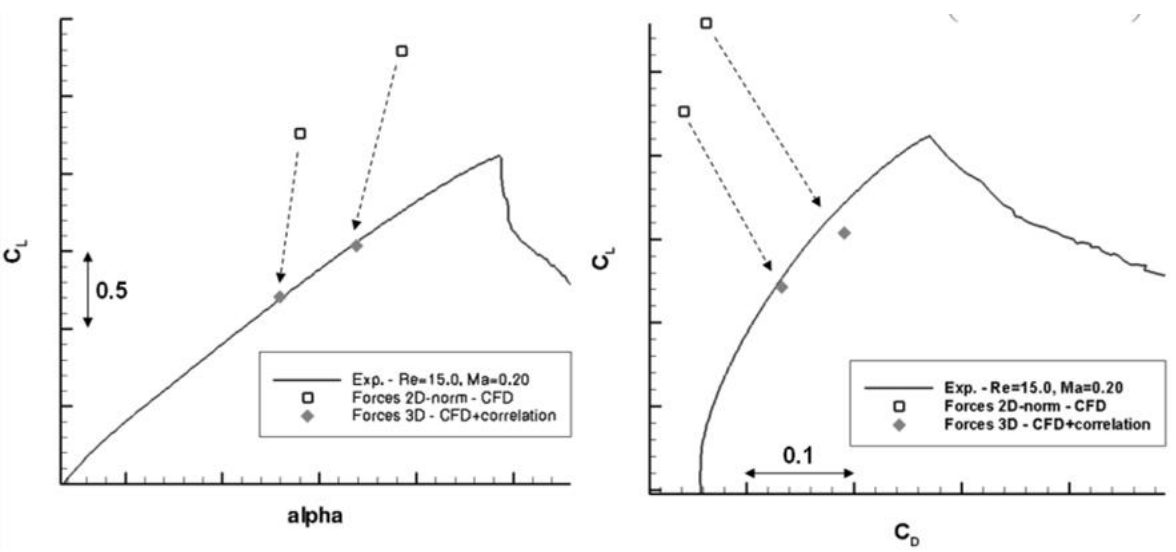

Figure 8: Comparison between computed 2D-normalized forces ( $\square$ ), forces computed through 3D correlation ( $\bullet$ of Eq.(14) and experimental data (solid line).

\section{SUMMARY OF THE EMPLOYED OPTIMIZATION TOOLS}

Within DeSiReH, a group of partners was appointed to solve the optimization problem illustrated in the above sections, using both 2D and 3D approaches, and by employing optimization and CFD capabilities currently available and routinely used at the time the project was launched. The consortium of partners was composed of:

- Industrial partners: Airbus-Germany (AI-D), Airbus-Military (AI-M), Piaggio Aero Industries (PAI);

- Research centers: Italian Aerospace Research Centre (CIRA), German Aerospace Research Centre (DLR), French Aerospace Research Centre (ONERA), and ;

- Universities: University of Padua (UNIPD).

Within the following subsections some information will be provided about the tools and strategies employed by partners to carry out the optimization, nevertheless without going into details about numerical settings used either in the CFD computations or in the setup of the optimization algorithms employed. As a matter of fact, all the participating partners have a large experience with both the optimization and CFD tools employed, therefore it is assumed that all the numerical setup chosen were calibrated to achieve the best accuracy, reliability and efficiency of the optimization chain. Nevertheless, some more details on individual setup can be found in $[25][26][27][28][29]$.

\section{H. Employed CFD tools and their calibration}

Table 1 summarizes the calibrated partners' CFD tools and some salient feature of the relevant numerical settings used. As shown, different meshing and CFD approaches (i.e., multiblock structured, hybrid and Chimera) were used, as well as both commercial and in-house developed software. As suggested in [22], a CFD tools calibration phase was firstly performed to avoid a large spreading of partners' results at the end of their 
optimization work. Before performing any optimization work, each partner has assessed its CFD setup (e.g., solver settings, meshing strategy/density, turbulence model, 2D angle of attack wherever applicable, etc.) by comparison with the available experimental data.

Table 1: Calibrated partners' CFD tools successively employed for optimization.

\begin{tabular}{|c|c|c|c|c|c|c|}
\hline Dimension & Partner & Solver & Grid Generator & Grid Type & Grid size & Turb. model \\
\hline $2 \mathrm{D}$ & AI-D & $T A U$ & In-house CENTAUR & 2D Hybrid & 135 kNodes & $\mathrm{k}-\omega \mathrm{MSST}$ \\
\hline $2 \mathrm{D}$ & UNIPD & ANSYS Fluent & ANSYS Gambit & 2D Structured & $220 \mathrm{kCells}$ & $k-\omega \mathrm{SST}$ \\
\hline $2 \mathrm{D}$ & CIRA & ZEN & ANSYS ICEMCFD/Hexa & 2D Structured & $100 \mathrm{kCells}$ & $\mathrm{k}-\omega \mathrm{TNT}$ \\
\hline $2 \mathrm{D}$ & DLR & FLOWer & In-house $M E G A C A D S$ & 2D Structured & $90 \mathrm{kCells}$ & SA (Edwards) \\
\hline $2.5 \mathrm{D}$ & ONERA & els $A$ & In-house MESH 3D & 2.5D Chimera & 88.5 kCells & SA \\
\hline $2.5 \mathrm{D}$ & AI-M & ANSYS CFX & ANSYS ICEMCFD/Hexa & 2D Structured & $100 \mathrm{kCells}$ & $k-\omega$ MSST \\
\hline $3 \mathrm{D}$ & AI-M & ANSYS CFX & ANSYS ICEMCFD/Hexa & 3D Structured & 2.5 MCells & k- $\omega$ MSST \\
\hline $3 \mathrm{D}$ & PAI & Metacomp CFD++ & ANSYS ICEMCFD/Hexa & $\begin{array}{l}\text { 3D Unstruct. } \\
\text { (hexa) }\end{array}$ & 2.8 MCells & k- $\omega$ SST \\
\hline
\end{tabular}

Several 2D meshing approaches used by partners are shown in Figure 9, together with a comparison of pressure distributions after tools calibration, for the TO-DP1 flow condition of the optimization problem described in $\S \mathrm{B}$. It is worth noting that in Figure 9 (left) also the partners' calibrated incidence is indicated. As discussed in $\S \mathrm{F}$ above, due to the missing sectional forces measurements in the $3 \mathrm{D}$ experiment, the $2 \mathrm{D}$ tools calibration was performed by considering only a best fitting of the experimental sectional pressure distribution available. However, as shown in the figure, the agreement of all partners' results versus the experiment is very satisfactory and the scatter of partners' pressure distributions is very small. The same calibration was performed for all other design points defined in $§ \mathrm{~B}$.
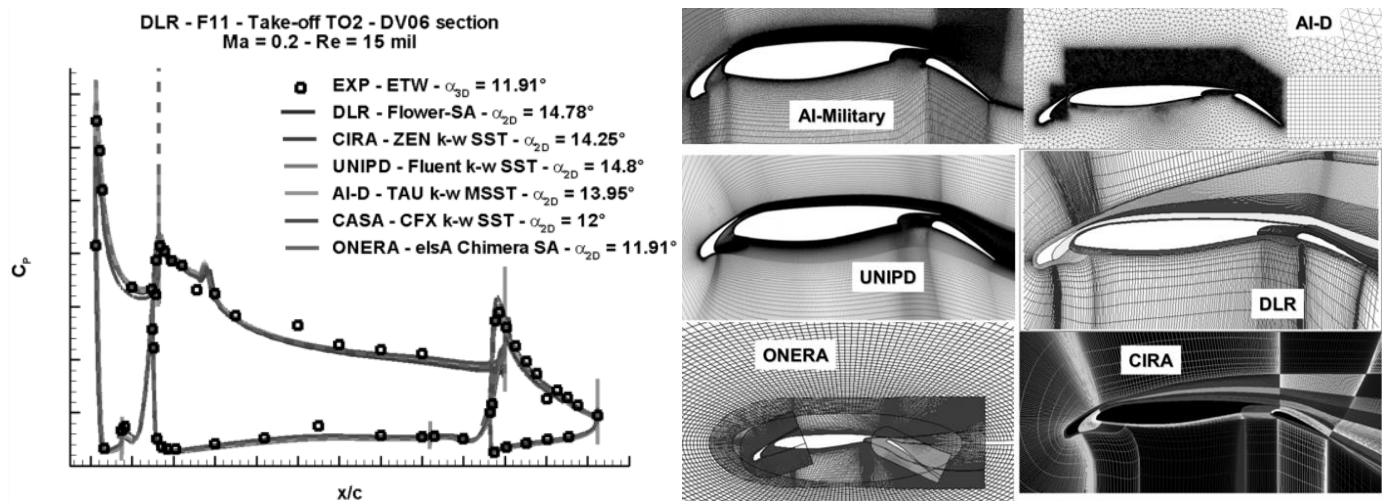

Figure 9: Example of 2D CFD tools calibration vs. experimental data (left) and 2-dimensional mesh generation approaches by partners (right). 
The 3D multiblock structured meshing approach by both PAI and AI-M is shown in Figure 10, together with a comparison of sectional pressure distributions at several wingspan locations, obtained at TO-DP1 flow conditions. For the $3 \mathrm{D}$ calibration activities also aerodynamic forces $\left(C_{L}\right.$ and $\left.C_{D}\right)$ were used to assess both partners' numerical setup and this, as it will be shown in the following, it will result in a reduced scatter of optimization results.

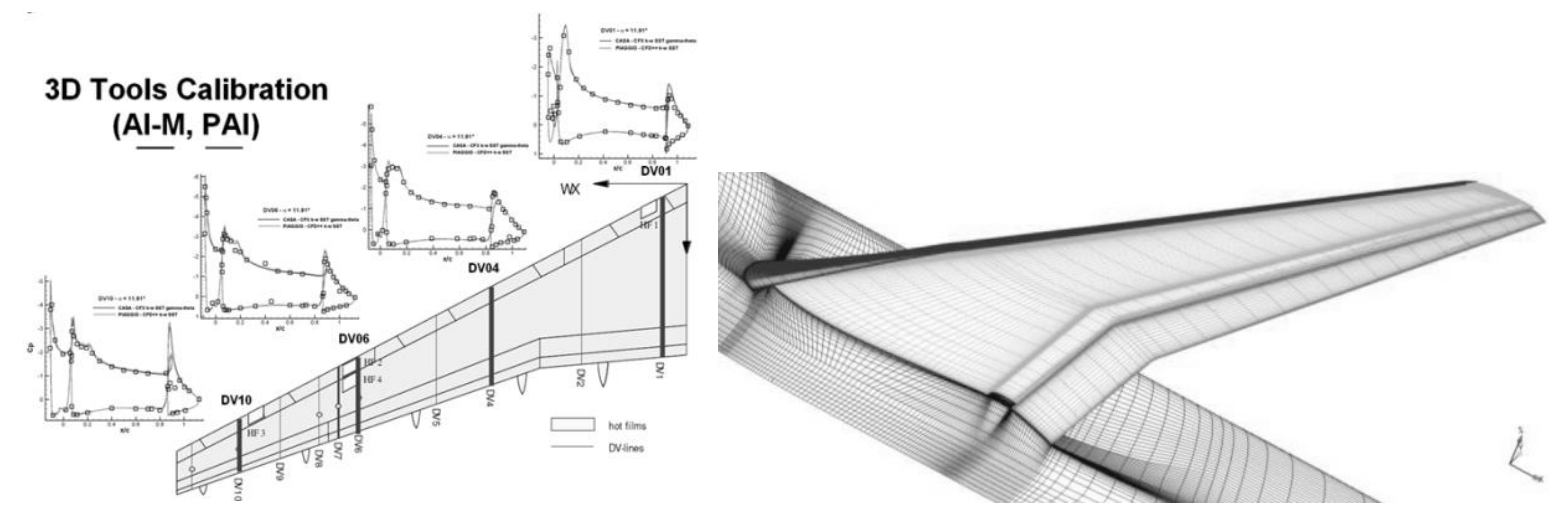

Figure 10: Example of 3D CFD tools calibration vs. experimental data (left) and some details of the 3D structured multiblock mesh generation approach by PAI/AI-M (right).

\section{Shape parameterization, optimization strategies and employed algorithms}

Table 2 presents an overview of the adopted shape parameterizations and software, optimization approaches, number of design variables (DVs) used, optimization algorithms and software. It is worth noting that 6 DVs are needed in 2D to completely define the slat (3 DV) and flap (3 DV) positioning at each flight conditions (i.e., 6 DV for TO and 6 DV for LDG).

Table 2: Summary of employed flow model, shape parameterization approach and SW used, optimization approaches, optimization algorithm and SW used. Number of DVs employed are in ( ) parentheses.

\begin{tabular}{|c|c|c|c|c|c|c|}
\hline Partner & $\begin{array}{l}\text { Flow } \\
\text { model }\end{array}$ & $\begin{array}{c}\text { Shape } \\
\text { parameterization }\end{array}$ & Shape SW & Optimization approach & Opt. Algorithm & $\begin{array}{c}\text { Optimization } \\
\text { SW }\end{array}$ \\
\hline AI-D & $2 \mathrm{D}$ & No shape optimization & - & Sequential: $\mathrm{TO}(6) \rightarrow \mathrm{LDG}(6)$ & Best engineering practice & None \\
\hline ONERA & $2.5 \mathrm{D}$ & Bézier curves (14) & $\begin{array}{l}\text { Fortran } \\
\text { program }\end{array}$ & Sequential: $\operatorname{LDG}(20) \rightarrow \mathrm{TO}(6)$ & CMAES & $D A K O T A$ toolkit \\
\hline AI-M & $2 \mathrm{D}$ & $\begin{array}{l}\text { Overposition of modes } \\
\text { (3) }\end{array}$ & $\begin{array}{c}\text { Matlab }^{\circledR+} \\
\text { ICEMCFD }^{\text {TM }}\end{array}$ & Sequential: $\mathrm{LDG}(9) \rightarrow \mathrm{TO}(6)$ & $\begin{array}{c}\text { LDG: Simplex+GA } \\
\text { TO: GA }\end{array}$ & In house SW \\
\hline DLR & $2 \mathrm{D}$ & $\begin{array}{c}\text { Free-Form } \\
\text { Deformation (5) }\end{array}$ & $\begin{array}{l}\text { In-house } \\
M E G A C A D\end{array}$ & Cumulated Obj: TO+LDG (17) & Rowan's Subplex & In house Pyranha \\
\hline CIRA & $2 \mathrm{D}$ & $\begin{array}{l}\text { Overposition of modes } \\
\qquad(16)\end{array}$ & $\begin{array}{l}\text { In-house } \\
\text { wg2Aero }\end{array}$ & Bi-Objective: TO \& LDG (28) & Genetic algorithm & In-house $G A$ \\
\hline
\end{tabular}




\begin{tabular}{|c|c|c|c|c|c|c|}
\hline AI-M & $3 \mathrm{D}$ & No shape optimization & $\begin{array}{c}\text { Matlab }^{(\circledR)}+ \\
\text { ICEMCFD }^{\text {TM }}\end{array}$ & Single Obj: $\mathrm{TO}(6)$ & Genetic algorithm & In house SW \\
\hline PAI & $3 \mathrm{D}$ & $\begin{array}{l}\text { Conic curves }+c \text {. } \\
\text { splines }(4)\end{array}$ & CATIA $^{\mathrm{TM}}$ v 5 & Single Obj: TO(11) & Genetic algorithm & CIRA's $G A$ \\
\hline
\end{tabular}

In the $3 \mathrm{D}$ case the number of DVs needed to describe a slat or flap positioning depends on the parametrization approach chosen. As shown, different shape parameterization approaches were adopted, leading to a different overall number of DVs employed in the optimization. It is worth remarking that most of the partners employed a considerable amount of parallel resources to carry out the optimization work.

\section{2 D approaches}

Regarding the $2 \mathrm{D}$ activities, the most simplified case is represented by the AI-D approach, wherein shape optimization was not considered at all and the only DV are those relevant to HL devices positioning. Moreover, as reported in [28][29], both ONERA and UNIPD employed a shape parameterization based on Bézier curves, though using a different number of DV. Similarly, both AI-M and CIRA employed a strategy based on overposition of modes for shape modification purposes. In particular, as reported in [25], CIRA used both HicksHenne and Rear Loading shape modification functions, while AI-M considered only Hicks-Henne functions leading to a reduced number of shape design variables. Finally, DLR employed 5 shape parameters for flap and slat shape description, by adopting the so called Free Form Deformation approach. For a summary of the aforementioned parameterization techniques, see [30].

One of the objectives of this work was to evaluate different optimization strategies for the design of HL systems. It is worth noting that the partners used their currently available optimization tools routinely used. A group of partners (AI-D, AI-M and ONERA) considered a decoupling of the objectives and split the original problem into two sequential, single-objective sub-problems (e.g., LDG first and TO afterwards), characterized by a reduced design space, whereas the other partners considered the bi-objective problem as a whole (CIRA and UNIPD) or using a cumulative function (DLR) (See Table 2).

- $\quad$ AI-D considered its routinely used hand-made optimization strategy, based on a successively refined parametric study of the elements' settings, where no shape optimization is included. As mentioned in $\S \mathrm{D}$, in this case the two objectives are not linked and two independent optimizations could be run for improving the TO objective first and LDG afterwards.

- $\quad$ AI-M modified the LDG objective (drag minimization) into lift maximization at LDG-DP1, since lift improvement is a more interesting target in military applications. AI-M reduced the original problem into 
several small sized sequential sub-problems, wherein each adverse flow mechanism is controlled by a reduced number of parameters. The starting assumptions of AI-M optimization process are:

- In order to achieve maximum lift at DP1, the flap deflection has to be maximized while maintaining either attached or only slightly separated flow on flap upper surface. Therefore, the flap deflection is not treated as an independent DV but is gradually increased up to a maximum allowed by onset of massive separation which prevents further lift increase;

- At low incidences (DP1), the limiting phenomena for increasing flap deflection is flap trailing edge separation. This separation can be controlled mainly by optimization of flap position;

- At high incidences (DP2), and at relatively low Mach number, the off-surface separation in the slat/wing wake is the critical phenomenon that limits the achievable lift. This separation is related to the development and interaction of the boundary layer on main element and slat wake. The most efficient way to reduce the off-surface separation with fixed flap deflection is a slat position optimization;

- It is also assumed that slat position has only minor effect on lift generated at low incidences (DP1) and flap position has a low importance at high incidences (DP2). Due to this assumption flap and slat were optimized separately.

According to such assumptions AI-M optimization process worked as follows. For a given flap deflection, the flap position is firstly optimized (2 DV) to either suppress any flap separation or to maximize the lift at DP1. The second step is to check the lift constraint at DP2, and if needed the optimization of slat position (in difficult cases also deflection can be included) is considered (2-3 DV) to suppress any off-surface separation. In case that either the lift increase (i.e., flap separation suppression) at DP1 or the lift constraint satisfaction (i.e., offsurface separation suppression) at DP2 is not successful, the process is restarted with a lower flap deflection. Otherwise a further flap deflection is considered and the process is repeated. Such an approach involves the usage of a very small number of DV in each sub-optimization loop (i.e., $2 \div 3 \mathrm{DV}$ ), and it is therefore easy to get a rapid convergence of the optimization algorithm employed (within 15 iterations in each sub-loop). The LDG settings optimization just described was performed by employing the Nelder-Mead (also known as Downhill Simplex or Amoeba method [31]) and afterwards only shape optimization (3 DV) was considered at LDG by using a Genetic Algorithm (GA). The new shape obtained was then frozen and a pure settings optimization was considered for TO, by using a GA. The GA is a real coded one and it implements typical operators like selection, elitism, crossover and mutation. Finally, worth to note that specific flow sensors were 
introduced for a fast and easy detection of the adverse flow phenomena mentioned above (i.e., flap separation, off-surface separation).

- ONERA considered two alternative sequential optimization strategies. The first one is a by element optimization, wherein first slat (settings and shape) optimization was performed at TO and LDG conditions with a frozen flap position (e.g., baseline). Next, the slat shape and position were frozen and a similar optimization at TO and LDG conditions was considered for the flap device. Such an approach demonstrated to be unsuccessful for the current (basically drag reduction) optimization problem, though it is known to be an effective strategy in lift maximization problems. Subsequently, an alternative by configuration sequential approach has been considered by ONERA, whose results are presented herein and detailed in [29]. In this case, first the slat and flap (settings and shape) were optimized at LDG, then the shape was frozen and both slat and flap settings were optimized at TO. In this last approach it was assumed that the shape DVs affect mainly the LDG performance. Such a sensitivity information was attained by post-processing the by element optimization results, which demonstrated anyhow to be useful in acquiring a pre-knowledge of the problem. The by configuration optimization has been carried out by using a so-called constrained Covariance Matrix Adaptation Evolution Strategy (CMAES), as detailed in [29].

- Furthermore, DLR considered a single-objective, multi-point optimization problem for a cumulative objective function (i.e., weighted sum of TO and LDG objectives), since a real independent multi-objective optimization was not possible at that time. In this case the size of the design space is not reduced, but the optimization problem is re-formulated as a single-objective problem (which will converge toward a specific point of the bi-objective Pareto front). The recently developed optimization suite Pyranha [34] was used by DLR, which offers a state-of-the-art python-based optimization environment including commonly used optimization strategies. For the specific problem at hand, the SUBPLEX method of Rowan [33] was selected, since it gives a good compromise between robustness, efficiency and performance. Four different optimization runs were carried out at DLR by considering: original bi-objective function, TO only, LDG only and $C_{L, \max }$ optimizations, respectively. It is worth noting that a coarser mesh size was used by DLR with respect to the one used for calibration (i.e., approximately $22.5 \mathrm{kCells}$ instead of $90 \mathrm{kCells}$ ), to carry out the optimization work.

A third group, composed by UNIPD and CIRA, implemented the original bi-objective/multi-point optimization problem and solved it the way it was formulated by considering 22 and 28 DVs, respectively. 
- CIRA used an in-house developed multi-objective genetic algorithm implementing the elitism mechanism to carry out the optimization work [25][27][32]. For the specific bi-objective optimization case at hand, an additional criterion based on a weighted average of objectives was introduced to define the best element and for implementation of the elitism mechanism. A preliminary optimization was carried out using a medium grid size (22.5 Kcells) and by identifying proper weights for the quadratic penalty functions adopted to account for constraints violations [25]. Next, the Pareto front of the medium grid optimization was used to partially fill the initial population of the fine grid optimization, in order to speed up the convergence of the GA.

- UNIPD used a state-of-the-art in-house multi-objective evolutionary algorithm for handling the optimization problem. Moreover, a surrogate model of the real fitness function coupled with a gradient-based algorithm was used during the evolution process to speed up the convergence rate and get improved solutions while reducing computational time. Specifically, the optimization algorithm used belongs to the family of the Surrogate-Assisted Memetic Algorithms (SAMA), whose main advantage over concurrent strategies lies in creating a synergy between global and local search: in particular, a GA is used for global search, while the local search is carried out using a gradient-based algorithm on a surrogate model of the objective function. The surrogate model was built using a feed-forward Artificial Neural Network trained by means of a BackPropagation algorithm. The transformation from a general multi-objective problem to a constrained singleobjective one was necessary for the implementation of the gradient-based algorithm for local search inside the SAMA framework.

\section{3D approaches}

As already specified in $\S \mathrm{D}$, the partners working with $3 \mathrm{D}$ approaches were requested to consider only the flap optimization for the TO conditions. Concerning the parameterization strategies used, AI-M worked with a pure setting optimization and no shape parameterization was considered, although also the 3D slat optimization was included in the process. On the other hand, PAI considered a simple flap shape parameterization based on conical curves and cubic splines (Figure 11). The advantage of such approach, extensively illustrated in [26], is the reduced number of DVs (only 4) employed to get a satisfactory description of the full 3D flap shape such as that considered herein. Moreover, Table 2 shows that both partners employed a Genetic Algorithm though using different strategies. In particular, AI-M considered a slat/flap setting only optimization, wherein the positioning of each element was specified using three independent variables: horizontal and vertical displacement and element deflection. The displacement variables were defined as relative to local chord. Constant relative displacement 
requires different absolute movement at element root and element tip. Due to this fact, the element displacement has to be realized as a sequence of linear motion in stream wise (say $x$ ) and vertical (say $z$ ) direction, and rotation about both axis (element roll and yaw).

PAI used a CAD-in-the-loop optimization approach [26], by modifying only flap shape and settings and involving globally $11 \mathrm{DVs}$. A fully parametric $3 D C A D$ model for the reference take-off configuration was developed using Dassault Systemes ${ }^{\oplus}$ CATIA v5 R19 SP2. During the optimization process, CATIA v5 was run in batch mode, provided with a dedicated routine (VB script macro) to read design variables values from an input text file, update the $C A D$ model, and export the updated geometry for subsequent use by the grid generator. The wing flap was designed in the retracted position, and then extended for take-off. Its shape was developed based on five different design sections: wing root, kink, tip, and two intermediate sections for the outboard wing. Independent flap segments were designed for the inboard and outboard parts of the wing, sharing the same design section at the wing kink.
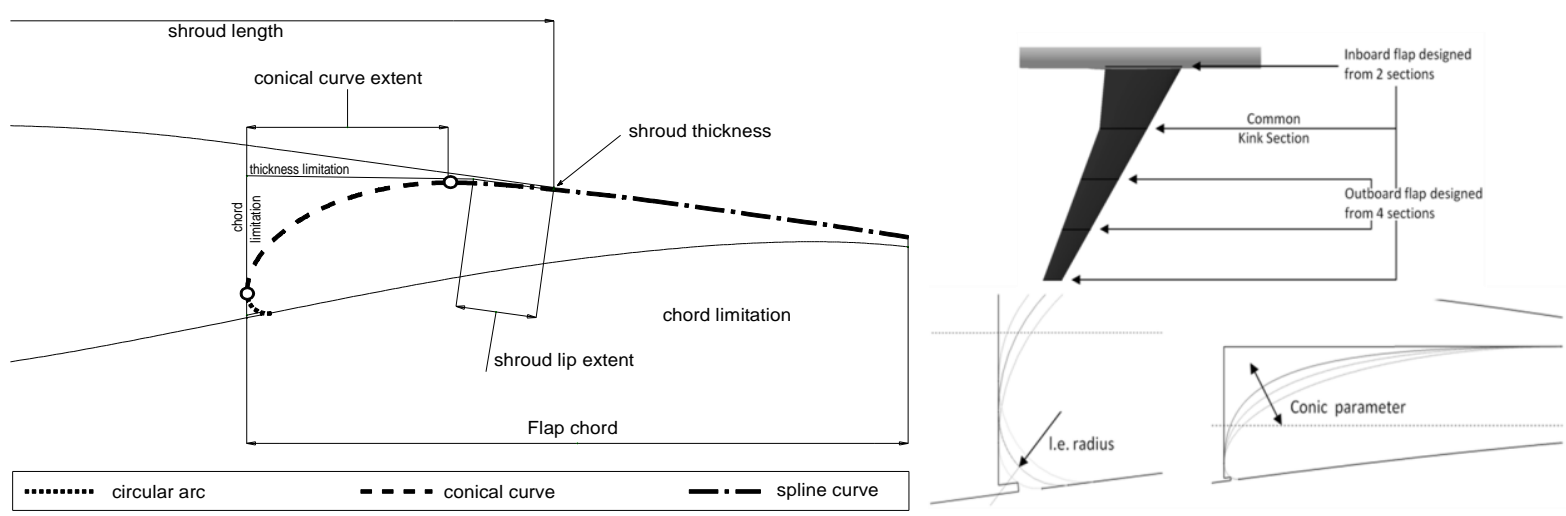

Figure 11: Flap shape parameterization (left) and 3D design sections (right) used by PAI.

During the conceptual definition phase of flap shape parameterization, the priority was set on the reduction of global design variables number. This is the reason why three different curves, with simple mathematics but no curvature continuity at their interfaces, were used for flap section design, instead of a single Bezier or B-Spline curve (Figure 11). A circular arc, tangent to the clean wing airfoil lower-side and to the flap chord limit bound, is first defined. Then a conical curve segment is used to connect the circular arc to a specified location on the flap thickness limit bound, preserving tangency continuity at both extremities. At the end, a cubic spline curve is drawn from the rearmost conic extremity, through the lower-side shroud lip corner, down to the upper-side trailing edge point of the local clean wing airfoil. Therefore, the flap shape at each design section is fully defined by the values of 4 independent parameters: 1 - circular arc radius; 2 - conical curve parameter; 3 - conical curve extent; 4 - shroud lip extent (directly related to the flap thickness). Parameter values for flap chord, shroud thickness and length, were not modified during the optimization. 
For the inboard flap segment, the extended position is achieved with a $2 D$ movement, which means that the trajectories of any flap body point, during its rigid motion, are planar and parallel to the same reference plane (Figure 12). The normal direction of this plane is defined by two angular parameters, which are free to be modified by the optimizer. Once the reference plane is identified, another three variables are necessary for the inboard flap setting to be fully defined: two scalar components of the planar translation and the rotation angle around the normal direction. Four different orthogonal left-handed reference frames are introduced to control the inboard flap deployment:

RF1. coordinates system with origin at the leading edge of flap root section, y-axis on the line connecting the leading edges of flap root and kink sections (in the retracted position) and $\mathrm{x}$-axis parallel to wing planform reference plane;

RF2. obtained with an Euler Z-X-Z rotation of the first reference frame around its origin, using only the first two Euler angles (the $\mathrm{XZ}$ plane of this system defines the reference plane of the 2D flap movement);

RF3. obtained by translating the second frame along its $x$ and $z$ axes (flap in-plane translation);

RF4. obtained by rotating the third reference frame about its $y$ axis (flap deflection).

The extended inboard flap position is therefore obtained using a coordinate transformation of the retracted flap geometry from axes \#2 to axes \#4. The outboard flap segment is instead extended with a fully $3 D$ movement. Only five of the six independent parameters are nevertheless necessary to define take-off settings, as the other one would produce span wise translations of the outboard flap. Four reference frames are defined, in a similar way as for the inboard flap, with initial origin at the leading edge of flap tip section. The extended position is in this case obtained using a coordinate transformation of the retracted flap geometry from axes \#1 to axes \#4, thus accounting also for the two Euler rotations between reference frames 1 and 2, which produce "yaw" and "roll" rotations of the outboard flap, thus modifying the flap gap and overlap along the wingspan.

The abovementioned setting parameters represent a convenient choice to control flap extended position. Traditional setting specification in terms of gap, overlap and deflection can be easily obtained as an output once the flap elements are positioned based on these reference input parameters. The industrial tools employed by PAI and illustrated in Table 1 have been coupled to CIRA in-house developed optimization toolbox (GA), based on Genetic Algorithms. 


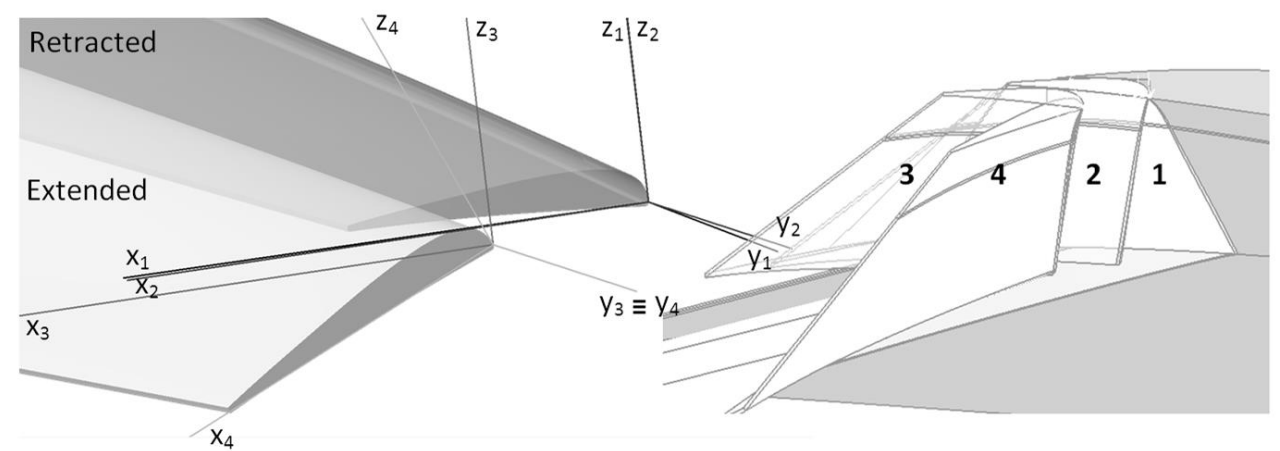

Figure 12: Outboard flap setting parameterization used by PAI.

\section{OPTIMIZATION RESULTS}

\section{J. 2D Results}

Table 3 shows the overall number of CFD evaluations performed by each partner (including both DP1 and DP2, wherever applicable) to achieve the complete optimization work, and in the last two columns also the number of evaluations spent for the TO and LDG objective improvements. The total number of DVs considered is also recalled and, for those partners employing sequential strategies, the typical number of DVs employed in suboptimizations is reported in parentheses. As it can be observed, the largest number of evaluations is associated to partners working with a larger number of DVs, as expected. Figure 13 presents the same information in terms of tendency curves. As shown, if the typical number of DVs is considered, the logarithmic tendency line provides a good approximation of the data and indicates that the number of DVs employed is the main responsible parameter for the overall number of evaluation needed for optimization. This is mainly demonstrated by comparing DLR, ONERA and UNIPD results, wherein, in spite ( $c f$. Table 2) of the different nature of optimization algorithms used (i.e. subplex, evolutionary, evolutionary + gradient on surrogate) the number of evaluations is comparable and increasing (logarithmically) with the number of DVs employed. Also, comparisons of ONERA, UNIPD and CIRA number of evaluations gives a sensitivity of the computational effort increase with the number of DVs adopted in the problem parameterization for comparable evolutionary optimization algorithms.

Table 3: 2D optimizations - Number of CFD evaluations performed by each partner, cumulative number of design variables considered, typical number of DVs employed in sub-optimizations (in parentheses), number of evaluations spent for TO and LDG objective improvements, respectively.

\begin{tabular}{ccccc}
\hline \hline Partner & $\begin{array}{c}\text { Nr of } \\
\text { CFD Evaluations }\end{array}$ & Nr of DV & TO evaluations & LDG evaluations \\
\hline AI-M & 521 & $15(3)$ & 210 & $101^{*}$ \\
AI-D & 978 & $12(6)$ & 528 & 450 \\
DLR & 1784 & 17 & 467 & 467 \\
ONERA & 3058 & $26(20)$ & 532 & 1262 \\
UNIPD & 3432 & 22 & 1716 & 1716 \\
CIRA & 7488 & 28 & 3744 & 3744 \\
\hline \hline
\end{tabular}




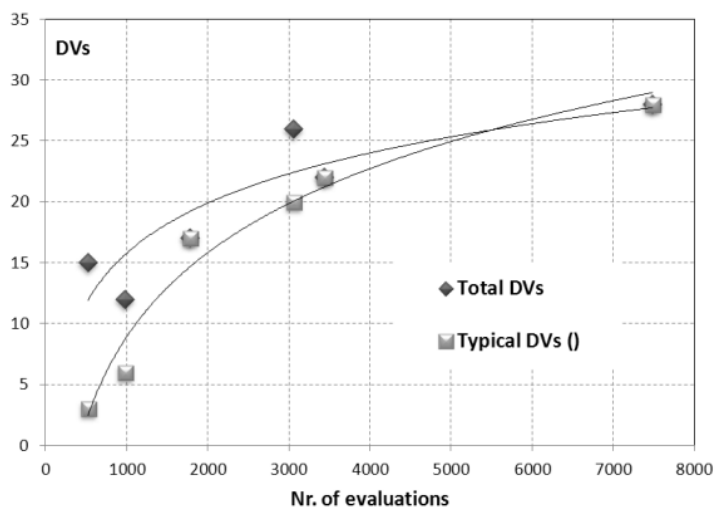

Figure 13: Tendency curves - Number of total/typical DVs employed vs. number of CFD evaluations.

\section{Comparison of optimized geometries}

The retracted shapes of the optimized configurations obtained by the different partners are presented in Figure 14 , showing that no clear "common design" trend stands out. It can be observed that all the designed shapes respect the geometrical constraints, and some partners' optimal solution show a little increase in the main element chord (ONERA and UNIPD). The deployed geometries of the optimized configurations found are presented in Figure 15 and in Figure 16 for LDG and TO conditions, respectively. Similarly to the retracted shapes, there is no clear common design that stands out about the elements' settings, excepted for the slat deflection and the flap gap at LDG. In fact, especially for the optimized LDG flap positions, it is shown that partners' solutions appear to fall into sub-groups of similar gap settings: a first group (AI-M, UNIPD, AI-D, CIRA) having a small flap gap, whereas a second group (DLR, ONERA) exhibits a larger and approximately the same gap as the baseline flap setting.
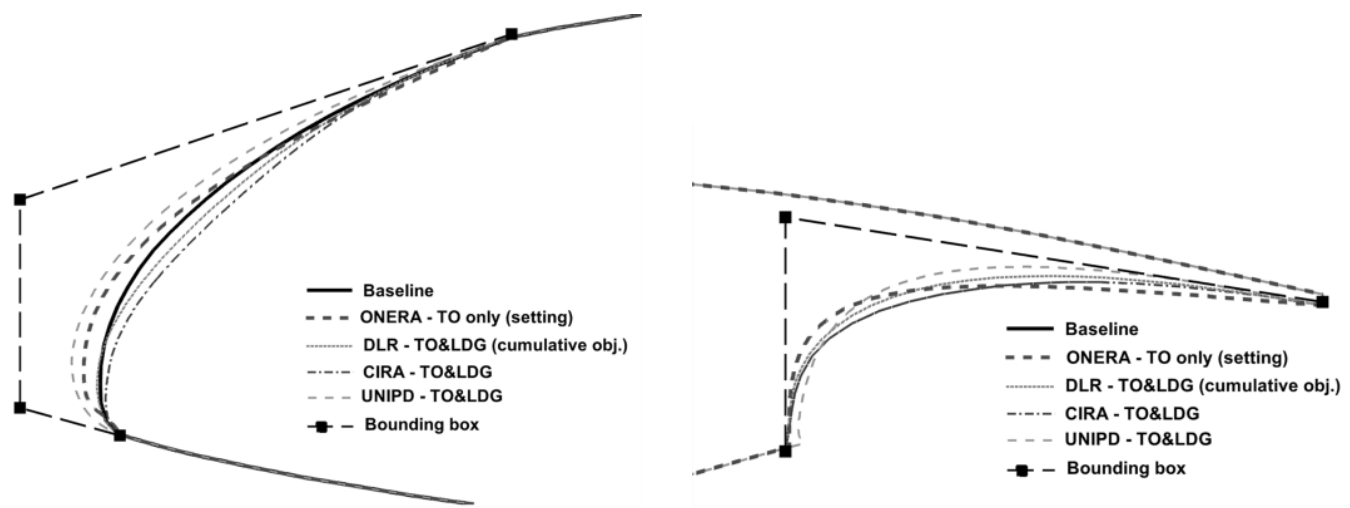

Figure 14: Partners' optimized shapes compared to the baseline configuration - Retracted airfoils with manufacturing bounding box constraints (dashed line). 

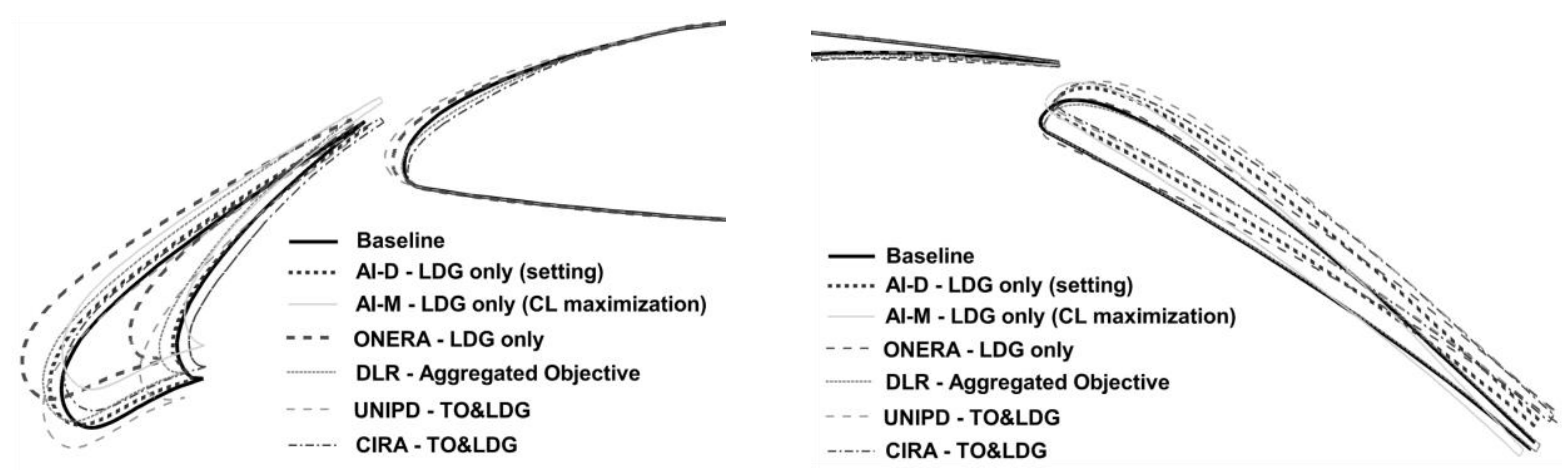

Figure 15: Optimized LDG configurations.

\section{Cross-comparison of $2 D$ results}

A cross-comparison of the optimized geometries produced was carried out by a restricted group of "evaluators", selected among the contributors: AI-D, ONERA, and UNIPD. Each evaluator used its own previously calibrated CFD method to predict the aerodynamic performance of the different optimized geometries $(c f$. $\S \mathrm{H})$. It is worth remarking that since the optimization problem statement defines design points in terms of a fixed 3D incidence, the $C_{P}$ calibration procedure $(c f . \S \mathrm{H})$ led to different values of $\alpha_{2 \mathrm{D}}$ for each partner.
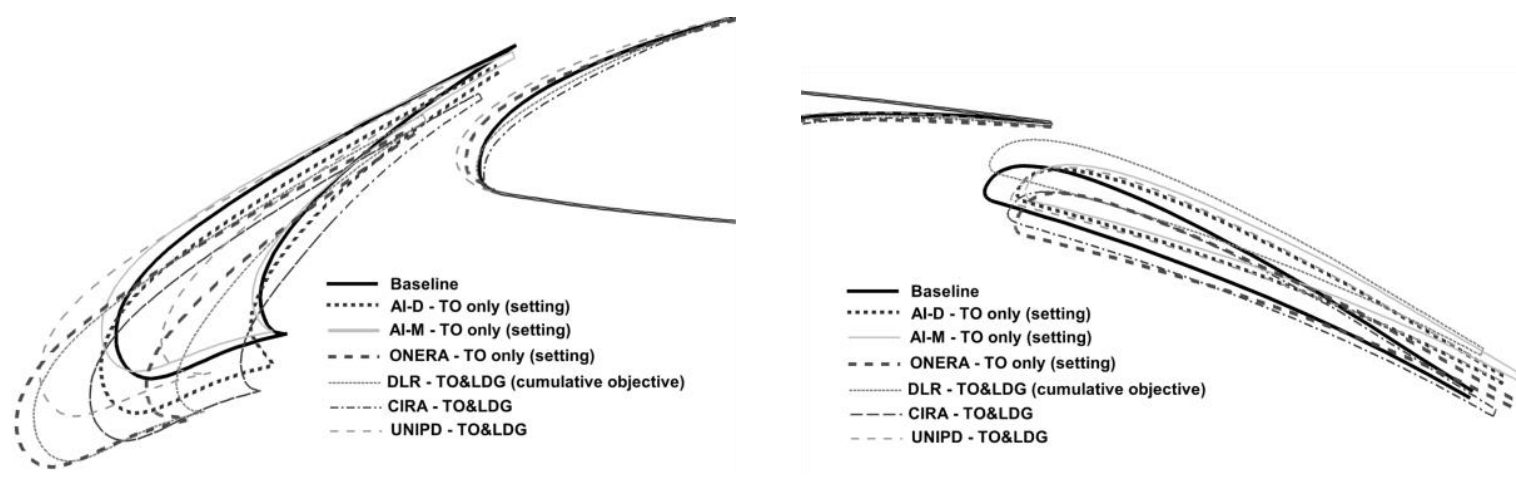

Figure 16: Optimized TO configurations.

Additionally, as it was previously observed ( $c f$. §D), both objective functions considered are directly (for LDG) or indirectly (for TO) dependent on the drag coefficient. Accordingly, depending on the calibrated $\alpha_{2 \mathrm{D}}$, each evaluator could estimate a lower/higher value of $\mathrm{C}_{\mathrm{L}}$ for a given configuration, which would have produced an artificial improvement/deterioration of the objective functions (and eventually also lift constraint violations). In order to overcome this problem and have a more rational comparison, the performance of the optimized configurations were performed by each evaluator at a constant lift level (i.e. at its own value of the baseline lift coefficient obtained in the calibration phase). Figure 17 presents the cross-checked results obtained by the evaluators at $\left.\left.C_{L}\right)^{\text {opt }}=C_{L}\right)^{\text {initial }}$ at both TO-DP1 (left) and LDG-DP1 (center). In this figure, on the $x$-axis is reported the optimized geometry delivered by each partner, whereas on the $y$-axis is reported the assessment 
performed by the three evaluators expressed in terms of relative improvement of objective function. Moreover, Figure 17 (right) shows, for each geometry assessed, the arithmetic average of the evaluators' judgment at TO and LDG. In the following, the AI-D improvements/efforts (obtained via standard industrial procedures based on "by hand" parametric studies and high human interaction) will be considered as a reference to assess the capabilities of automatic optimization tools.

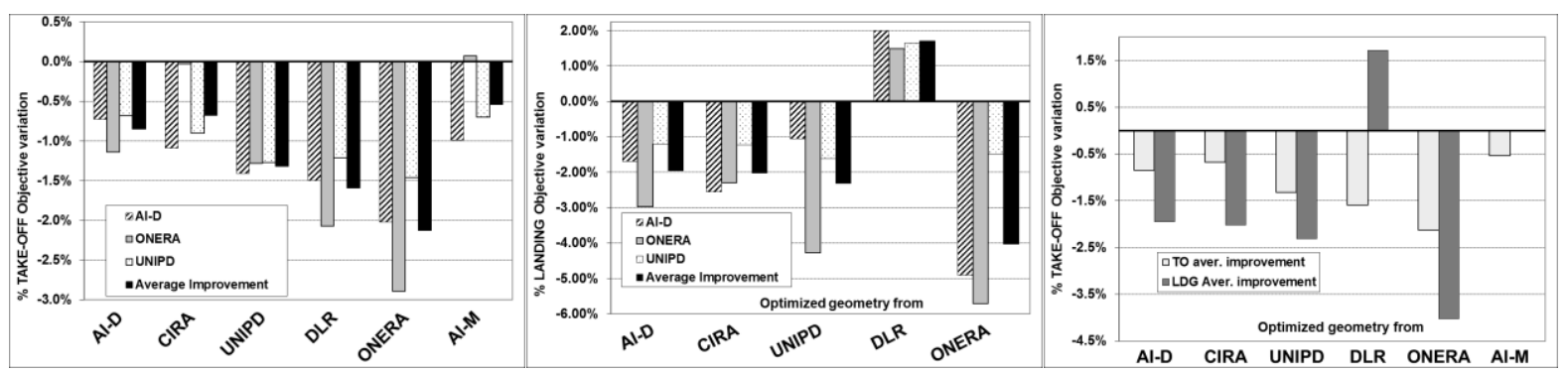

Figure 17: Crosscheck of results at $C_{L}=$ cost. for DP1 at TO (left) and LDG (center). Averaged evaluators' results at TO and LDG (right) for each geometry.

With reference to Figure 17 (center), we can firstly observe that all the evaluators found a degradation of the performance for the DLR configuration at LDG condition. This can be explained by the use of a too coarse grid during the optimization process $(22.5$ kcells $)$, which led to inconsistent results when the CFD evaluation is repeated using a finer mesh $(\approx 90$ kcells). However, from DLR-TO results, it appears that such a mesh density, thought inadequate for LDG optimization, did not exhibit the same inconvenient at TO. Moreover, CIRA and UNIPD obtained similar performance improvements using comparable approaches, but not a remarkable better performance over the AI-D approach, especially at LDG. On the other hand ONERA, working with a reduced design space (thanks to the problem decoupling), could more widely explore the design space in each of the singleobjective optimizations performed, probably explaining the larger improvements achieved for both objectives considered. It is worth noting that direct comparison of AI-D vs. ONERA results at LDG show the potentials of automatic optimization capabilities when a suitable strategy is considered. On average, the ONERA optimized solution led to an improvement of about $4 \%$ for LDG conditions and $2.15 \%$ for TO conditions, approximately the double of what achieved with the "by hand" parametric approach by AI-D.

Finally, Figure 18 presents the cross-checked performance of the AI-M ( $\mathrm{C}_{\mathrm{L}}$-optimized) geometry, confirming the achieved lift performance improvement obtained at fixed incidence. It is worth remarking that the poor performance of AI-M configuration at TO (Figure 17) could be justified by the few evolutions of the GA algorithm considered in the TO optimization phase (210 evaluations, $c f$. Table 3$)$.

Figure 19 compares ONERA optimum solution with the initial (baseline) configuration. Geometries are compared on the left-hand side of the figure, whereas on the right side are compared the 3D (extrapolated 
according to $\S \mathrm{G})$ aerodynamic performance of the two configurations, estimated through the calibrated ONERA CFD chain.

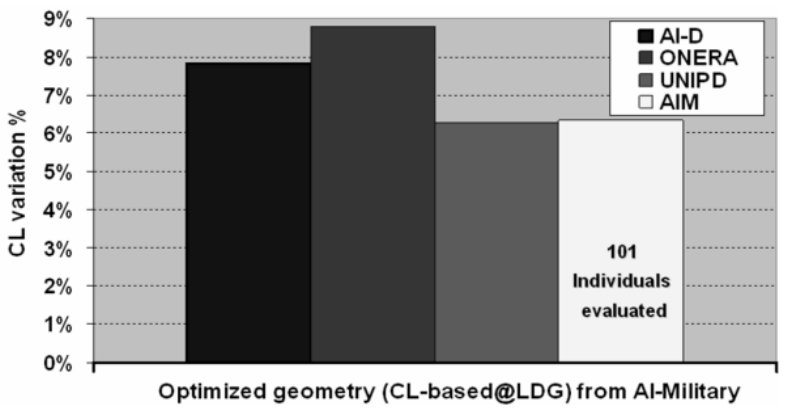

Figure 18: Cross-check of AI-M lift-based optimized geometry at LDG.

Figure 19 (top-right) shows that at LDG conditions the lift constraints are satisfied at both design points and a drag coefficient reduction is achieved at DP1 (and kept over the whole $C_{L}$ range). Similarly, Figure 19 (bottomright) also shows that the lift constraints at both design points are satisfied and the TO objective function is reduced in a large range of incidences till $\alpha_{D P 2}$. Additionally, it can be observed that the additional off-design post-checks on lift are satisfied together with (although not reported herein) the constraints on pitching moments and minimum drag at LDG. Concerning the geometry changes, this drag minimization problem leads to a decrease of the flap deflection for both LDG and TO configurations.
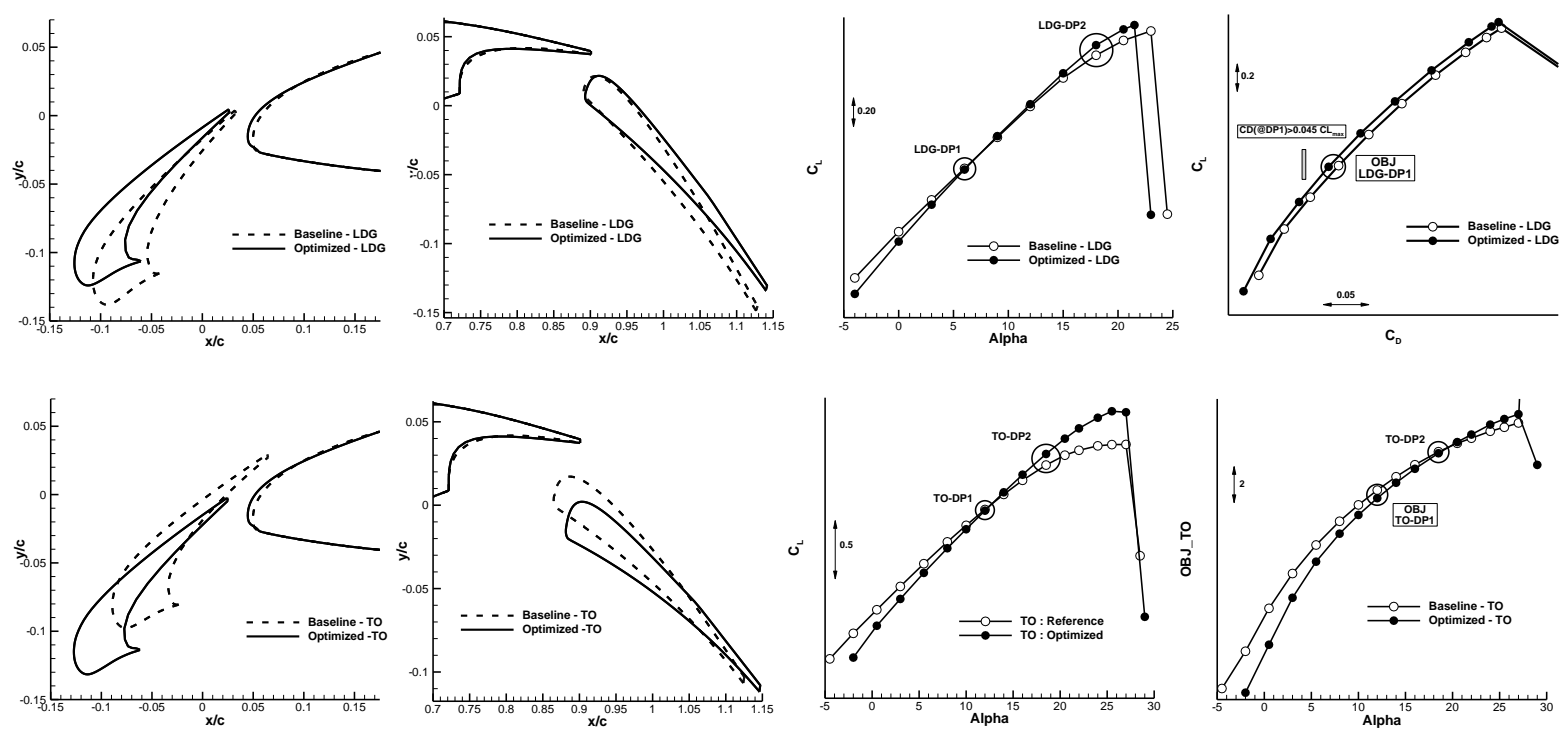

Figure 19: Comparison of ONERA optimal vs. baseline geometries (left) and their LDG (right-top) and TO (right-bottom) performance (3D-extrapolated values).

For the LDG problem, the changes in slat and flap elements are nearly equivalent to a single rotation (around the slat trailing-edge or the flap leading-edge). For TO problem, the slat deployment leads to a decrease in overlap, with gap and deflection around their initial values. For the flap, the optimization leads to significant modification of settings compared to the initial values (increase of gap and decrease of overlap and deflection). 
Due to the heterogeneous set of approaches employed and to the specific problem considered, exhibiting quite a flat objective functions, it is not possible to draw definitive conclusions on the best optimization strategy and algorithm to employ in HL optimization. Nevertheless, the experience gained was useful to provide the following general guidelines for HL design and suggested areas for future investigations:

- For the same decoupled approach, the automatic ONERA optimization provided double improvements compared to the AI-D approach based on current industrial standards. This indicates that the introduction in the aerospace industry of automatic optimization for HL design purposes has a big potential, as it would allow for interesting margins of performance improvements. Nevertheless, other partners' results have shown that automatic optimization does not always produce clear improvements over the industrial approach. This happens especially when the design space is not adequately explored (either because the design space is too wide or because the optimization is stopped prematurely).

- The observation of CIRA/UNIPD/ONERA-LDG optimization data indicates that the usage of a high number of DVs requires a large number of evolutions to achieve significant improvements in evolutionary approaches (convergence). In this context, a comparison of CIRA vs. UNIPD performance indicates that usage of surrogate models can help convergence enhancement, although another viable method might be to use more simplified shape parameterizations (e.g., the 3D PAI approach described in $\S \mathrm{H})$ to reduce the design space extent. Finally, ONERA-LDG optimization results (using a comparable number of DVs as CIRA/UNIPD) indicate a potential superiority of the employed Covariance Matrix Adaptation Evolution Strategy algorithm over the more classical evolutionary approaches employed by CIRA and UNIPD. Altogether, this indicates the potential interest of using adjoint methods, but these methods are not mature yet for high-lift applications [1].

- $\mathrm{AI}-\mathrm{M}$ (LDG) physics-driven optimization, being based on a very small number of DVs proven to be very powerful and cost-effective (only 101 evaluations required). As expected, the introduction of engineering knowledge allows efficient formulation/solution of the problem and it is always suggested whenever possible.

- The DLR experience indicates that strategies based on approximate fitness function evaluation (e.g., a coarse grid) are to be carefully employed in HL numerical optimization and that preliminary assessments are needed to setup a reliable strategy. In other words, the quality of flow solution used by optimizer is of higher importance compared to computing costs.

- The usage of decoupling/sequential strategies (allowing a reduced design spaces) is highly recommended. However, not all the sequential strategies are effective. As mentioned above, the "by element" decoupling 
considered by ONERA resulted to be unreliable for the current drag-based problem, whereas the "by configuration" sequence shown herein demonstrated to be very useful. This indicates that applicability of sequential strategies must be assessed a-priori by preliminary analyses or pre-knowledge of the problem.

\section{K. 3D Results}

Both PAI and AI-M used a 3D multiblock structured mesh, initially generated by PAI and employed in the calibration phase illustrated in $\S \mathrm{H}$, by implementing their own mesh parameterizations. Moreover, it is remarked that PAI focused on the flap shape/setting optimization, whereas AI-M focused on the flap and slat setting only optimization.

PAI considered CIRA's in house developed GA software [32], coupled to the routinely used industrial CAE tools, as described in $§ \mathrm{IV}$ and more detailed in [26]. A population of 12 individuals was evolved for 82 generations during the single-objective (TO) optimization, using a random initialization around the baseline, $100 \%$ standard binary crossover, a-posteriori elitism and 3\% mutation probabilities. Lift and pitching moment constraints were included into the design by means of quadratic penalties. Appropriate values for the quadratic penalty weights have been derived in [25], based on a sensitivity analysis of the objective function with respect to lift and drag coefficients at DP1. The evolution over 82 generations ( 2000 CFD simulations) is shown Figure 20. The best individual over the whole history shows a total improvement of about $0.43 \%$ in the objective function, and most of the reduction (about $0.35 \%$ ) is achieved after the first 25 generations. Though not shown here in details, the PAI-CIRA optimum configuration slightly failed to satisfy the DP1 lift constraint and therefore the objective improvement includes a small amount of penalty. The PAI-CIRA optimization process considered geometry and mesh updates on a domestic performance machine using a single CPU and a total of 4GB RAM, whereas the CFD simulations were run in parallel on 72 processors of a computing cluster with $3 \mathrm{GHz}(1333 \mathrm{MHz}$ FSB) peak performance cores. The average elapsed time per individual evaluation was about 50 minutes (20 minutes per CFD run, 4 minutes each for CAD update and mesh generation, 1 minute for data transferring), the time per generation was about 10 hours and the overall elapsed time for optimization was about 35 days.

AI-M employed a conventional, real coded genetic algorithm developed in house and assumed a population size of 50 individuals, the Latin Hypercube technique for initialization, 98\% crossover (49\% uniform and $49 \%$ arithmetic), $2 \%$ a-priori elitism and 5\% mutation. The optimization process was run for only 7 generations (700 CFD simulations) due to limitations with the available computational resources. The performance improvement obtained by AI-M is approximately $0.12 \%$ with respect to the initial configuration. The total elapsed time for one 
CFD run was about 1.5 hour and a total of 1050 hours (44 days) have been spent for the optimization task on a 24 core cluster of similar performance as the PAI one.

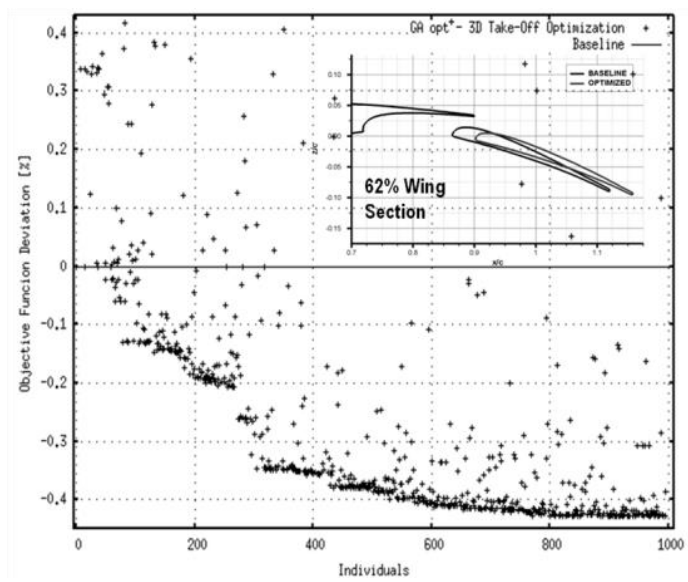

Figure 20: Convergence history of PAI-CIRA 3D optimization.

According to the different optimizations considered by both partners (i.e. PAI flap only vs. AI-M flap and slat), a cross-comparison is performed only in terms of the achieved target performance improvements. With this aim, in order to have a fair comparison and to make the assessment clear of small constraints violations (due to different CFD setup and treatment of constraints, as highlighted in $\S \mathrm{J})$, PAI recomputed both optimal geometries at $\left.\left.C_{L}\right)_{T O-D P 1}^{o p t}=C_{L}\right)_{T O-D P 1}^{\text {initial }}$, similar to the approach followed in $\S \mathrm{J}$. Moreover, the cross-check was carried out on a finer mesh (6.8 Mcells), solving the turbulent boundary layer down to the wall on both wing and fuselage (instead of the wall-function approach used with the PAI optimization mesh). Table 4 shows that the objective function improvement levels claimed by the partners were confirmed also on the finer mesh level (thought with some small variations), with the PAI-CIRA configuration providing the highest objective function reduction. At these testing conditions the PAI-CIRA configuration allows a reduction of 21 drag counts, versus the 7 drag counts reduction achieved by the AI-M configuration. Furthermore, both partners respected the pitching moment constraint, with the AI-M configuration providing higher pitching moment than the PAI-CIRA one, though without achieving any remarkable benefit in terms of target improvement. Finally, the post-optimization check on lifting capabilities close to maximum lift conditions (i.e., eq. (4)) shows that PAI-CIRA configuration very slightly violated this lift constraint by one lift count (0.01).

Table 4: Comparison of the 3D optimized TO configurations from AI-M and PAI-CIRA, computed on a 6.8Mcells CFD mesh $\left(1 \mathrm{drag}\right.$ count $\left.=10^{-4}\right)$.

\begin{tabular}{|c|c|c|c|c|}
\hline \multirow[b]{2}{*}{ FINE MESH@DP1 } & \multicolumn{3}{|c|}{$C_{L}=C_{L, \text { initial }}$} & \multirow{2}{*}{$\begin{array}{c}\alpha=19 \mathrm{deg} \\
\Delta \mathrm{C}_{\mathrm{L}}\end{array}$} \\
\hline & $\begin{array}{c}\text { Total } \Delta \mathbf{C}_{\mathbf{D}} \\
\text { (drag counts) }\end{array}$ & $\% \Delta$ Obj-TO & $\% \Delta \mathrm{C}_{\mathrm{M}}$ & \\
\hline OPTIMIZED AI-M & -7 d.c. & $-0.16 \%$ & $-11.4 \%$ & +0.01 \\
\hline OPTIMIZED PAI-CIRA & -21 d.c. & $-0.44 \%$ & $-1.0 \%$ & -0.01 \\
\hline
\end{tabular}


Figure 21 illustrates a comparison between PAI-CIRA optimized configuration and the baseline, in terms of both geometries and sectional pressure distributions (the reference section is that considered in the $2 \mathrm{D}$ optimizations). As shown, the design evolved towards an increase of flap gap, a reduction of the overlap and (although not clear visible) limited shape modifications mainly addressing flap thickness reduction and leading edge radius increase. Moreover, the pressure distribution indicates the tendency of the optimizer to increase the aerodynamic load on the flap element while decreasing it on the main wing, thus providing an overall equal total lift.

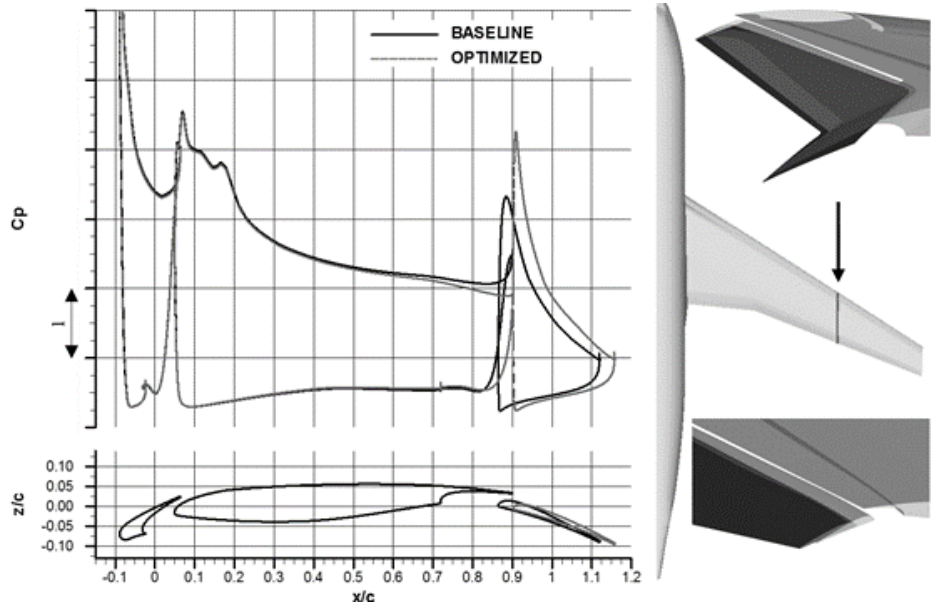

Figure 21: Comparison of Baseline vs. 3D optimized configuration from PAI-CIRA. Geometries and sectional pressure coefficient distributions at $62 \%$ half-span section, DP1 conditions (left). 3D flap positioning (right).

The effect of such a load swap can be explained with the help of Figure 22, wherein a Mach number contour plot around both configurations is shown. As visible, the main effect of unloading the main wing in the optimized configuration is a weaker main wing wake with respect to the baseline, therefore producing a reduced viscous drag contribution with respect to the baseline. As a counterpart, the higher flap load on the optimized configuration produces a slightly stronger flap wake (and a higher boundary layer thickness at flap trailing edge), therefore an increased flap viscous drag contribution is expected with respect to the baseline, though the overall balance is in favor of the wing viscous losses reduction.
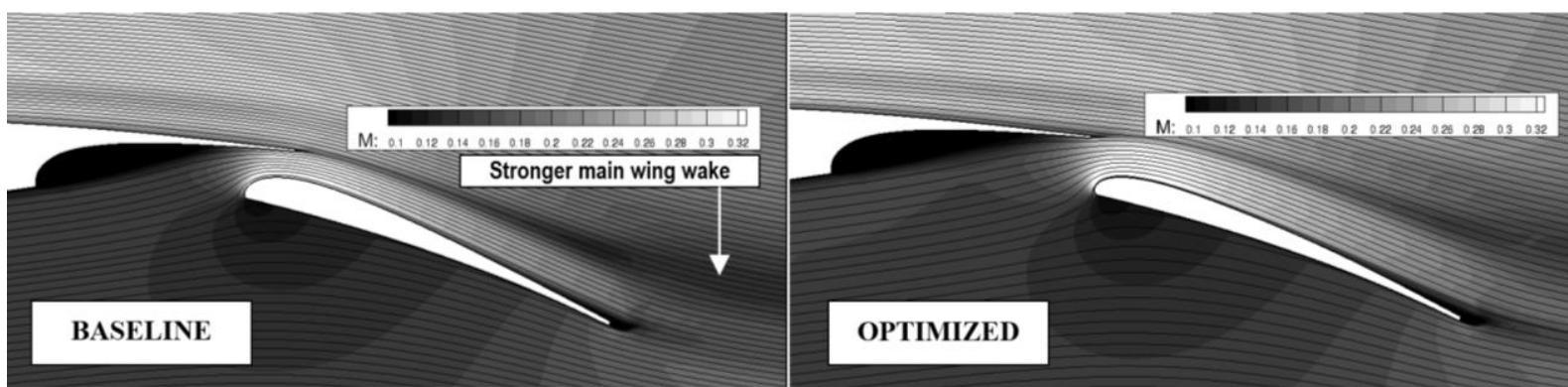


\section{Figure 22: Comparison of Baseline vs. 3D optimized configuration from PAI-CIRA. Mach contours at $62 \%$ half-span section. DP1 conditions.}

\section{CONCLUSIONS AND FUTURE PERSPECTIVES}

A concurrent multi-objective/multi-point high-lift design activity was targeted in the DeSiReH project, aimed at the comparison of state-of-art optimization tools capabilities. The optimization problem was formulated in such a way to include, as much as possible, the most salient features encountered in industrial applications (such as structural and manufacturability constraints). Moreover, a proper procedure already used and demonstrated in [22] was adopted to correlate the $2 \mathrm{D}$ results to the $3 \mathrm{D}$ wing performance, together with a suitable strategy for high-lift elements' deployment in 2D and a calibration procedure. Herein, the several partners involved challenged their design tools and adopted different decoupling strategies, shape parameterizations, CFD tools, turbulence modeling, meshing approaches and optimization algorithms. The experience gained from this exercise highlighted that the introduction of automatic optimization tools for HL design purposes has a big potential in comparison with a human based approach. In fact, while the automatic approach allowed treating, in a relatively easy way, the complex constrained multi-objective problem characterized by a large design space, the human based approach required the introduction of several simplifications at the cost of reduced improvements achieved. Nevertheless, in section $\mathrm{V}$ it was shown that automatic optimization does not always produce clear improvements over the industrial approach, especially when the design space is not adequately explored. In principle, the most efficient way to solve the proposed problem is, whenever possible, to adopt decoupling strategies and to introduce engineering based knowledge and physics-driven optimization criteria to reduce the design space size. According to the involved industrial partners' view, the industry perspective is the need for a robust HL optimization tools, rather than on finding the optimum with unnecessary high precision (e.g. squeezing out the very last drag-count). A robust framework is needed, which can handle multiple objectives, various constraints, and a large number of design parameters in an efficient optimization environment. In this context, the evolutionary algorithms seem to be an appealing tool for HL design, although it was highlighted the drawback of massive CFD evaluations required to achieve significant improvements. Though this problem is counterbalanced with the huge and increasing HPC capabilities nowadays available at aircraft manufacturers, efficiency remains a key issue in the future employment of these tools. To this aim, both the usage of surrogate models and simplified/approximate shape parameterizations seems to be key areas of research and development, which can help convergence and efficiency. Also, smart formulations of both constraints and objective functions might keep some potential for efficiency increase. Although a lead-time reduction compared to today's standard HL design processes is not yet visible, a potential 
in terms of improved performance is evident. Future improvements in the areas mentioned above can result in less design loops, reducing the overall project lead-time and possibly reducing the amount of wind tunnel entries.

Two industrial partners, PAI and AI-M, have demonstrated the applicability of automatic 3D optimization for high lift configurations, by coupling optimization tools to routinely used industrial CAE tools. For the problem considered the improvements found were fairly small for both partners in comparison to the computational efforts spent, one could judge them as negligible. This was mainly justified by the flatness of the objective function considered, as indicated in section $\mathrm{D}$, but also because the starting configuration was already representative of an earlier good industrial design. However, the scope of this activity was the demonstration of how to setup such a complex 3D optimization framework in an industrial context, and to perform a robust multipoint shape and elements' setting optimization with it. The big benefit being that a larger amount of design parameters can be handled at once, in a completely automatic way and without human work. Additionally, the industrial appraisal of the application rises further when multi-disciplinary constraints (e.g. structural constraints, kinematic feasibility, etc.) are included in the optimization problem. Finally, thanks to the fully automated process and the evolutionary approach, several high lift concepts can be evaluated in parallel, which allows the designer to down select from a larger variety of devices in comparison with the manual approach.

\section{Acknowledgments}

This work has been performed within the scope of the DeSiReH project [23] (Design, Simulation and Flight Reynolds Number testing for advanced High Lift Solutions), funded by the European Commission within the $7^{\text {th }}$ Framework Program, grant number ACP8-GA-2009-233607. The authors also wish to thank other contributors to the results shown herein: Zbynek Hrncir (Airbus-Military), Henning Strüber (Airbus-Deutschland) and Timo Kühn (DLR). Finally, the authors wish to thank Dr. Jochen Wild from DLR, coordinator of the DeSiReH project, which contributed to the statistical analysis shown in $\S \mathrm{C}$ and the scaling laws illustrated in $\S$ III.

\section{References}

[1] A. Flaig, R. Hilbig: “High-Lift Design for Large Civil Aircraft." High-Lift System Aerodynamics, $71^{\text {st }}$ Fluid Dynamics Panel Meeting and Symposium, Banff (Canada) 1992. AGARD CP-515, Sept. 1993, pp 31-1-3112.

[2] C.P. van Dam: “The Aerodynamic Design of Multi-Element High-Lift Systems for Transport Airplanes.” Progress in Aerospace Sciences, Vol. 38, No. 2, 2002, pp. 145-180, doi:10.1016/S0376-0421(02)00003-9. 
[3] P.K.C. Rudolph: "High Lift Systems on Commercial Subsonic Airliners." NASA Contractor Rept. 4746 , Sept. 1996.

[4] P.T. Meredith: "Viscous phenomena affecting high-lift systems and suggestions for future CFD development." AGARD CP 315, 1993, pp. 19-1-19-8.

[5] J. Perraud, J. Cliquet, R. Houdeville, D. Arnal, F. Moens: “Transport Aircraft Three-Dimensional High-Lift Wing Numerical Transition Prediction.” Journal of Aircraft, Vol. 45, N5, 2008, pp. 1554-1563. doi:10.2514/1.32529.

[6] A.M.O. Smith: "High-Lift Aerodynamics." Journal of Aircraft, Vol. 12, No6, 1975, pp. 501-530. doi:10.2514/3.59830.

[7] C.L. Rumsey, S.X. Ying: "Prediction of High Lift: Review of Present CFD Capability." Progress in Aerospace Sciences, Vol. 38, No 2, 2002, pp. 145-180. doi:10.1016/S0376-0421(02)00003-9

[8] D.A. King, and B.R. Williams: “Developments in Computational Methods for High-Lift Aerodynamics." The Aeronautical Journal, Vol. 92, № 917, 1988, pp. 265-288.

[9] K. Kusunose, L. Wigton, P. Meredith: “A Rapidly Converging Viscous/Inviscid Coupling Code for Multielement Airfoil Configurations.” AIAA Paper 91-0177, 1991.

[10] M. Drela: “Newton Solution of Coupled Viscous/Inviscid Multielement Airfoil Flows.” AIAA Paper 90-1470, June 1990.

[11] T. Cebeci: “Calculation of Multielement Airfoils and Wings at High Lift: High-Lift System Aerodynamics.” High-Lift System Aerodynamics, $71^{\text {st }}$ Fluid Dynamics Panel Meeting and Symposium, Banff (Canada) 1992, AGARD CP 515, Sept. 1993, pp. 24-1-24-15.

[12] S. Eyi, K.D. Lee, S.E. Rogers, D. Kwak: "High-Lift Design Optimization Using the Navier-Stokes Equations. ”27 $7^{\text {th }}$ Fluid Dynamics Conference, AIAA Paper 1996-1943, June 1996.

[13]E. Besnard, A. Schmitz, E. Boscher, N. Garcia, T. Cebeci: “Two-Dimensional Aircraft High Lift System Design and Optimization." 36 ${ }^{\text {th }}$ AIAA Aerospace Sciences Meeting and Exhibit, AIAA Paper 98-0123, 1998.

[14] S. Kim: “Design Optimization of High-Lift Configurations Using a Viscous Adjoint-Based Method.” Ph.D. Dissertation, Stanford Univ.,Palo Alto, CA, 2001.

[15]M. Nemec, D.W. Zingg, T.H. Pulliam: "Multi-Point and Multi-Objective Aerodynamic Shape Optimization." $9^{\text {th }}$ AIAA/ISSMO Symposium on Multidisciplinary Analysis and Optimization, AIAA Paper 2002-5548, Sept. 2002. 
[16] H. Nakayama, H.-J. Kim, Matsushima, K. Nakahashi, K. Takenaka: “Aerodynamic Optimization of MultiElement Airfoil." 44 ${ }^{\text {th }}$ AIAA Aerospace Sciences Meeting and Exhibit,AIAAPaper 2006-1051, Jan. 2006.

[17]D. Yiju, D. Zhuoyi, Z. Hao: “Aerodynamic Optimization of Multi-Element Airfoils by Genetic Algorithms.” West-East High Speed Flow Field Conference, Moscow, Russia, Nov. 2007.

[18]E. Onate, G. Bugeda, T. Zhili, and J. Periaux: "Multi-Element Airfoil Lift Maximization Problems with Uncertainties Using Evolutionary Optimization and Unstructured Meshes.” European Conference on Computational Fluid Dynamics, edited by P.Wesseling, E. Oñate, and J. Périaux, ECCOMAS, Barcelona, 2008 .

[19] Y.-T. Lee, V. Ahuja, A. Hosangadi, M. Ebert: "Shape Optimization of a Multi-Element Foil Using an Evolutionary Algorithm. ” Journal of Fluids Engineering, Vol. 132, Issue 5, May 2010, pp. 051401-051412. doi:10.1115/1.4001343

[20] R.M. Greenman, K.R. Roth: “Minimizing Computational Data Requirements for Multi-Element Airfoils Using Neural Networks.” Journal of Aircraft, Vol. 36, No 5, 1999, pp. 777-84.doi:10.2514/2.2533

[21]D. Quagliarella: “Airfoil Calculations in High-Lift Conditions Using the MAVIAN Program.” Centro Italiano Ricerche Aerospaziali, Technical Note CIRA-TN-98-124, Capua, Italy, Dec. 1998.

[22] J. Wild, J. Brezillon, O. Amoignon, J. Quest, F. Moens, and D. Quagliarella: “Advanced High-Lift Design by Numerical Methods and Wind Tunnel Verification Within the European Project Eurolift II. ” Journal of Aircraft, Vol. 46, № 1, Jan.-Feb. 2009, pp. 526-541.doi:10.2514/1.37148

[23] European Commission. 2012. Aeronautics and Air Transport Research $-7^{\text {th }}$ Framework Programme $2007-$ 2013. European Union. ISBN 978-92-79-14287-1. 34-36.

[24] P. Iannelli, M. Minervino, J. Wild, H. Strüber, F. Moens, A. Vervliet: “Design of a High-Lift System for a Laminar Wing." $5^{\text {th }}$ European Conference for Aeronautics and Space Sciences - EUCASS 2013 1-5 July 2013, Munich (Germany)

[25]P. Iannelli, D. Quagliarella: “Multi-objective/Multi-point Shape and Setting High-Lift System Optimization by Means of Genetic Algorithm and 2D Navier-Stokes Equations." EUROGEN 2011 Conference proceedings.

[26] M. Minervino, P. Iannelli, D. Quagliarella: “3D Flap design using Navier-Stokes equations and evolutionary optimization techniques on an industrial platform." EUROGEN 2011 Conference proceedings.

[27] D. Quagliarella and P.L. Vitagliano: "Evolutionary Optimization of the EUROLIFT II Take-Off Design Case.” EUROGEN 2005 Conference proceedings. 
[28] E. Benini, R. Ponza and A. Massaro: "High-Lift Multi-Element Airfoil Shape and Setting Optimization Using Multi-Objective Evolutionary Algorithms." Journal of Aircraft. Vol. 48. № 2. 2011. 683-696. doi: $10.2514 / 1.54038$

[29]F. Moens, C. Wervaecke: “Multi-Point Optimization of Shapes and Settings of High-Lift System by Means of Evolutionary Algorithm and Navier-Stokes Equations.” Engineering Computations (2013). Vol. 30. 4:601-622. doi: 10.1108/02644401311329398.

[30] J. A. Samareh: “A Survey of Shape Parameterization Techniques.” CEAS/AIAA/ICASE/NASA Langley International Forum on Aeroelasticity and Structural Dynamics (1999) 333-343.

[31] J. A. Nelder, R. Mead: “A simplex method for function minimization.” Comput. J.,(1965) 7. 308-313.

[32] A. Vicini, D. Quagliarella: “Inverse and Direct Airfoil Design Using a Multi-objective Genetic Algorithm." AIAA Journal. Vol. 35. Nº 9. Sept. 1997. 1499-1505.

[33] T. H. Rowan: “Functional Stability Analysis of Numerical Algorithms. PhD Dissertation.” The University of Texas at Austin, 1990.

[34] J. Brezillon, A. Ronzheimer, D. Haar, M. Abu-Zurayk, M. Lummer, W. Krüger, F.-J. Natterer: “Development and Application of Multi-Disciplinary Optimization Capabilities Based on High-Fidelity Methods." $8^{\text {th }}$ AIAA Multidisciplinary Design Optimization Specialist Conference. AIAA Paper 2012-1757, April 2012.

[35] S. Kleinveld, O. Amoignon: “Adjoint Methods in High-Lift Design Optimization.” $5^{\text {th }}$ European Conference for Aeronautics and Space Sciences - EUCASS 2013 1-5 July 2013, Munich (Germany). 